\title{
Solid Deposition in the ITER Cryogenic Viscous Compressor
}

\author{
Dongsheng Zhang ${ }^{1}$, Franklin K. Miller, and John M. Pfotenhauer* \\ Department of Mechanical Engineering \\ University of Wisconsin-Madison, \\ 1500 Engineering Drive, Madison, WI 53706 USA
}

\begin{abstract}
A transient model for the ITER cryogenic viscous compressor (CVC) is presented. The CVC is designed to separate hydrogen isotopes from helium in the gasmixture exhaust from the ITER torus. During their residence in the CVC, hydrogen isotopes are captured along the pump wall while helium flows through. The CVC thereby provides the first stage of helium compression. The transient model characterizes the transport phenomena (species, momentum, and energy) that occur in the CVC. The numerical results are compared with experimental data from a scaled down test of the ITER CVC using pure hydrogen. Although the model has been developed for a hydrogen-helium mixture, it is simplified here in order to compare with the experimental data. The transient model, along with other numerical models we have developed, provide guidance for the design and optimization of the ITER CVC. The model can also be a useful tool or a reference for similar analyses, such as those for cryogenic carbon capture and air ingress in vacuum isolated cryogenic vessels.
\end{abstract}

Keywords: Cryopump, adsorption, transport phenomena, numerical models, ITER

*Corresponding author: pfot@engr.wisc.edu

${ }^{1}$ Present address: Oracle, 5750 Hannum Avenue Suite 200, Culver City, CA 90230 


\section{Introduction: The ITER Cryogenic Viscous Compressor}

Due to its enormous size and complexity, ITER presents numerous challenges for handling the flow of gases both into and out of the toroid-al plasma region that represents the heart of its operation. A recent and thorough description of the various vacuum systems associated with these flows is provided by Pearce et al. [1]. As described therein, six "Torus Cryogenic Pumps" (TCP) are sequentially utilized to evacuate the neutralized gas and helium that are produced by the operating fusion tokamak. The molecules that leave the plasma are pumped in the TCPs by adsorption onto activated charcoal at $4.5 \mathrm{~K}$. Of the six TCPs included in the ITER design, only four of them are operating at any given time, while the other two undergo a regeneration procedure. During the regeneration process, a roughing pump system removes the released gases, including hydrogen isotopes, trace amounts of helium, and plasma enhancement gases such as neon or nitrogen, from the TCPs.

The cryogenic viscous compressor (CVC) along with mechanical pumps, make up the roughing system used to pump the fusion exhaust gas during the regenerating process of the TCPs [1-3]. The CVCs represent the first stage of the roughing pump system. They are designed to capture the hydrogen isotopes for subsequent reprocessing while allowing the trace helium to pass through. The CVC's unique working conditions present a number of significant modeling challenges. For example, the CVC behaves as a heat exchanger between the relatively warm low-pressure flow stream of the fusion exhaust gas, and the supercritical helium coolant. Condensation of the exhaust gas components at the CVC wall, or evaporation of the same components from the CVC wall, depends on the difference in pressure between the bulk gas flow and the saturation pressure associated with the wall temperature of the CVC. An energy balance at the wall that includes factors such as enthalpy in turn determines the wall temperature profile and phase change energies of the captured gas, heat transfer to the coolant, and a thermal resistance that depends on the thickness of mass deposited on the wall. Because of the interdependence of all these various factors, the operation of the CVC is very much a dynamic system.

In view of the complex nature of the CVC, both experimental and theoretical investigations have been carried out in order to characterize its operation and provide appropriate design guidelines. Oak Ridge National Lab has built and tested a scaled down model of the CVC [4-6], while efforts at the University of Wisconsin-Madison [7] have focused on the physical analysis and modeling. The results of the model display that the combination of conservation equations for mass (species), momentum, and energy allow one to determine the rate and location of mass deposition on the walls of the scaled-down $\mathrm{CVC}$, as well as the temperature and pressure profiles through its length.

The following sections will briefly review the experimental sub-scale CVC test at ORNL and subsequently describe in detail the development and results of an associated numerical model at UW-Madison. The experimental data provide a reference for the theoretical models, and the theoretical work can provide guidance for any future experiments and the operation of the full scale CVC. 


\section{Sub-scale CVC test at ORNL}

The scaled down version of the ITER cryogenic viscous compressor being tested at Oak Ridge National Lab exists as a concentric tube-in-tube heat exchanger, spanning a length of about $1.07 \mathrm{~m}$, with an inner diameter of $5 \mathrm{~cm}$, and an overall outer diameter of $15.24 \mathrm{~cm}$. Pure hydrogen gas, or a mixture of hydrogen isotopes and with trace amounts of helium gas (about 1\%), flows through the inner tube while cold helium gas $(7 \mathrm{~K}-9 \mathrm{~K})$ flows as a coolant through the outer annular tube.

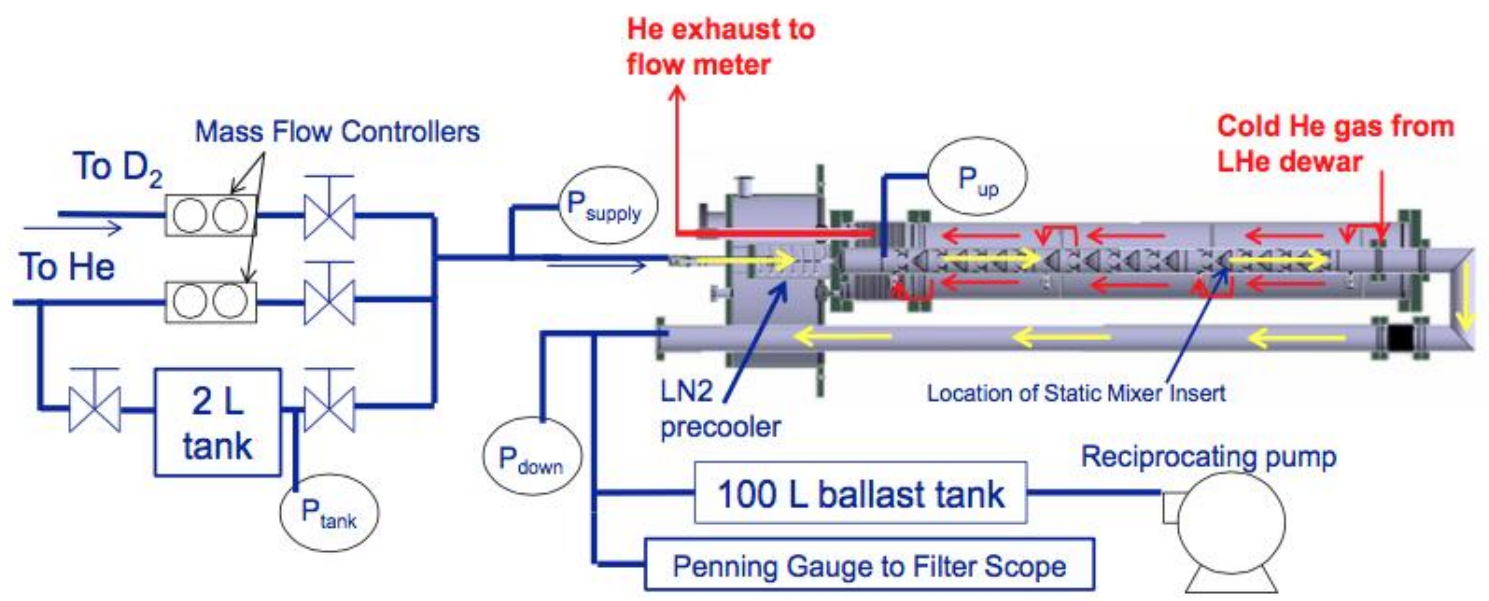

FIGURE 1a. Experiment setting for testing the single tube prototype CVC (courtesy of Oak Ridge National Lab).

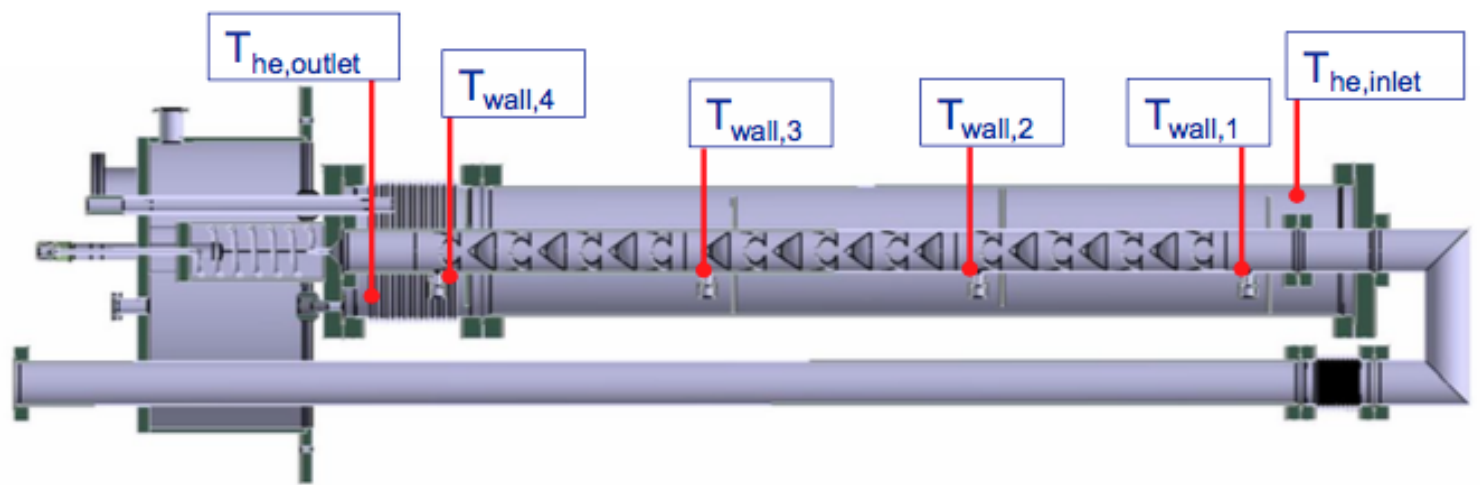

FIGURE 1b. Location of thermometers on the prototype CVC test rig (courtesy of Oak Ridge National Lab).

FIGURE 1a shows the experiment setup, including the concentric tube-in-tube heat exchanger, mass flow controllers, instrumentation, and roughing pump. As shown in FIGURE 1b, four silicon diode type thermometers, with a typical accuracy of less than $+/-30 \mathrm{mK}$ are mounted on the outer surface of the cryopump tube to measure the tube wall temperature. To avoid communication with the helium coolant flow stream, some insulation surrounds the temperature sensors. As measured from the hydrogen flow entrance (left) end, the first thermometer (measuring $T_{\text {wall, } 4}$ ) is located $10.2 \mathrm{~cm}$ axially along the tube. The three subsequent thermometers are mounted $30.5 \mathrm{~cm}$ apart along the 
tube, and they measure $T_{\text {wall, } 3}, T_{\text {wall,2, }}$, and $T_{\text {wall, } 1}$ respectively. Two silicon diode type thermometers are used to measure the inlet and outlet temperatures of the helium coolant. The thermometer measuring the helium coolant inlet temperature is mounted on a Teflon screw secured to a baffle and located about $7.5 \mathrm{~cm}$ axially beyond the $T_{\text {wall, }, 1}$ thermometer $(106.7 \mathrm{~cm}$ from the left end) and radially about $5 \mathrm{~cm}$ inside the outer wall. The thermometer measuring the helium coolant outlet temperature is mounted on a Teflon rod about $10.2 \mathrm{~cm}$ from the left end of the tube and secured with Teflon nuts approximately $2.5 \mathrm{~cm}$ radially inside the outer tube wall.

Two pressure gauges (MKS model 722B with accuracy of $0.5 \%$ of reading) are used to measure the upstream and downstream pressure of the hydrogen flow, and these provide absolute pressure measurements. The upstream pressure gauge is connected with a capillary tube to a location $7 \mathrm{~cm}$ from the left end of the tube, while the downstream pressure gauge is mounted on the exit of the entire setup. An axial distance of approximately $2.16 \mathrm{~m}$ separates the two pressure transducers. Note that the inner concentric tube extends beyond the co-axial section of the CVC tube in which region it has an inner diameter of approximately $5 \mathrm{~cm}$. The downstream pressure gauge is located at the outlet of this extended section. Additional pressure gauges are used to measure the pressure of the hydrogen gas supply and the ballast tank. The gauge for the hydrogen gas supply is mounted downstream of the flow controllers but upstream of the precooler.

Model 1480A MKS Instruments flow controllers are used as mass controllers. For hydrogen flow, the controller is a $0-10000 \mathrm{sccm}$ controller, while for helium flow, the controller is a 0-1000 sccm controller. Each of the controllers is calibrated for their respective gas. The controllers utilize a $0-5 \mathrm{~V}$ control signal and the control signal is adjusted to set the incoming flow rates.

The u-tube at the far-right end of the test rig has an outer diameter of $5.08 \mathrm{~cm}$ with a wall thickness of $0.9 \mathrm{~mm}$. Each leg of the u-tube is nominally $10 \mathrm{~cm}$ long and the connecting piece is approximately $15 \mathrm{~cm}$ long. The buffer volume of the ballast tank is approximately 30 inches long with a $40.6 \mathrm{~cm}$ diameter.

A helium coolant flow rate of about $0.5 \mathrm{~g} / \mathrm{s}$ is associated with a velocity of about $3 \mathrm{~mm} / \mathrm{s}$. If the tube length is divided into 50 nodes, the time for the helium coolant to travel from one node to its adjacent neighbor is about 8 seconds.

A hydrogen gas flow rate of about $0.001 \mathrm{~g} / \mathrm{s}$, is associated with an inlet velocity of about $4 \mathrm{~m} / \mathrm{s}$. The velocity decreases quickly as the gas is cooled.

It is helpful to note a variety of relevant time constants and length scales associated with the sub-scale CVC test rig: For the inlet condition of hydrogen flow at 80 $\mathrm{K}$ and $100 \mathrm{~Pa}$, the speed of sound is $733.1 \mathrm{~m} / \mathrm{s}$ and it thus takes about $0.001 \mathrm{~s}$ for a (viscous) pressure signal to travel the length of the $1 \mathrm{~m}$ cryogenic viscous compressor. The mass diffusion coefficient is about $0.01335 \mathrm{~m}^{2} / \mathrm{s}$, and it thus takes about $0.01 \mathrm{~s}$ for molecules to diffuse from the centerline to the surface of a tube with a $5 \mathrm{~cm}$ diameter.

At $80 \mathrm{~K}$, the thermal velocity of hydrogen molecules is about $916.6 \mathrm{~m} / \mathrm{s}$. At this temperature and for pressures less than $6 \mathrm{~Pa}$, hydrogen is rarefied and the molecules traveling at their thermal velocity will take about 0.0005 seconds to form 5 adsorption 
layers on the surface of the cryogenic pump. At $10 \mathrm{~K}$, the transition from viscous to rarefied conditions occurs as the pressure falls below $0.7 \mathrm{~Pa}$.

At $80 \mathrm{~K}$ and $100 \mathrm{~Pa}$, the mean free path of hydrogen and helium are about 0.0329 $\mathrm{mm}$ and $0.0523 \mathrm{~mm}$ respectively. Both are much smaller than the $50 \mathrm{~mm}$ pump diameter. When the gas temperature and pressure approaches $10 \mathrm{~K}$ and $0.7 \mathrm{~Pa}$, the mean free path becomes comparable to 0.01 times the pump diameter, the condition at which the transition from viscous to molecular flow begins. Note that the saturation temperature and associated saturation pressure at which the mean free path of hydrogen equals 0.01 times the pump diameter is $6.155 \mathrm{~K}$ and $0.458 \mathrm{~Pa}$.

The diffusion coefficient for hydrogen gas ranges between $0.0004 \mathrm{~m}^{2} / \mathrm{s}$ and 0.03 $\mathrm{m}^{2} / \mathrm{s}$, and depends significantly on temperature.

\section{Analysis and governing equations}

\subsection{Molecular and viscous flow regimes}

Typically, cryopumps are used to create or maintain a relatively high vacuum $[8,9]$, and usually operate in the rarefied gas regime. Molecules are 'pumped' when they are trapped on solid surfaces at cryogenic temperatures. The pumping process results from a physical adsorption mechanism that depends on factors such as surface condition and molecule species. As a result, a Monte Carlo method [10-12] is usually applied to model the physical processes operative in a cryopump.

In contrast with common cryogenic pumps, the nature of the ITER CVC is the cryogenic deposition of the hydrogen molecules from a viscous gas flow into the liquid or solid form on the pump surface. The crucial point is that the CVC works in the viscous flow regime. In fact, even if at the inlet temperature of $80 \mathrm{~K}$, the total inlet gas pressure is as low as $6 \mathrm{~Pa}$, the gas remains in the viscous, rather than the molecular flow regime. The incoming hydrogen molecules collide with other molecules more frequently than with the pump surface, and they behave as a bulk gas flow. Additionally the ITER CVC processes many more molecules than those being processed by a common cryogenic pump.

As a result, neither chemical adsorption nor physical adsorption is the major mechanism for cryogenic pumping in the ITER CVC. It is possible though that the gassurface interaction is important at the very beginning of the cryogenic pumping process if the pump surface is initially clean and free of contamination. The available sites for physical adsorption are too few compared to the large amount of hydrogen molecules entering the pump. The process of the gas-surface interaction is very short and unimportant in the entire cryogenic pumping process. The available sites are soon occupied, and the surface of the ITER CVC is quickly covered by the hydrogen molecules.

The ITER cryogenic viscous compressor differs from typical cryopumps in that it operates in the viscous flow regime and therefore the analysis associated with its operation requires a different modeling approach. To begin, one must consider the interaction between the gas comprising the bulk flow and the surface boundary. As mentioned in the introduction, condensation or evaporation will occur at the surface of the CVC if the pressure of the bulk flow is respectively higher or lower than the 
saturation pressure associated with the surface temperature. If the surface temperature is low enough, this equilibrium exchange of molecules between the gas and surface occurs via sublimation and/or de-sublimation. In either case, the exchange is accompanied with a phase change from vapor to liquid or solid, depending on the surface temperature.

For the CVC, in contrast with a cryopump, the adsorption rate is not determined by the molecular (thermal) velocity but by diffusion driven via the gradient in density, temperature, and pressure normal to the wall. The adsorption energy is the sum of the enthalpy difference between the molecules at the mean flow temperature and those at the phase equilibrium temperature, and the phase change energy, which depends on the phase equilibrium temperature at the wall. FIGURE 2 shows hydrogen sublimation energy as a function of temperature, plotted from published experimental data [13-14]. Compared with the energy of $1383 \mathrm{~J} / \mathrm{mol}$ released by cooling gaseous $\mathrm{H}_{2}$ from $80 \mathrm{~K}$ to $13.9 \mathrm{~K}$, the sublimation energy represents a significant addition of energy.

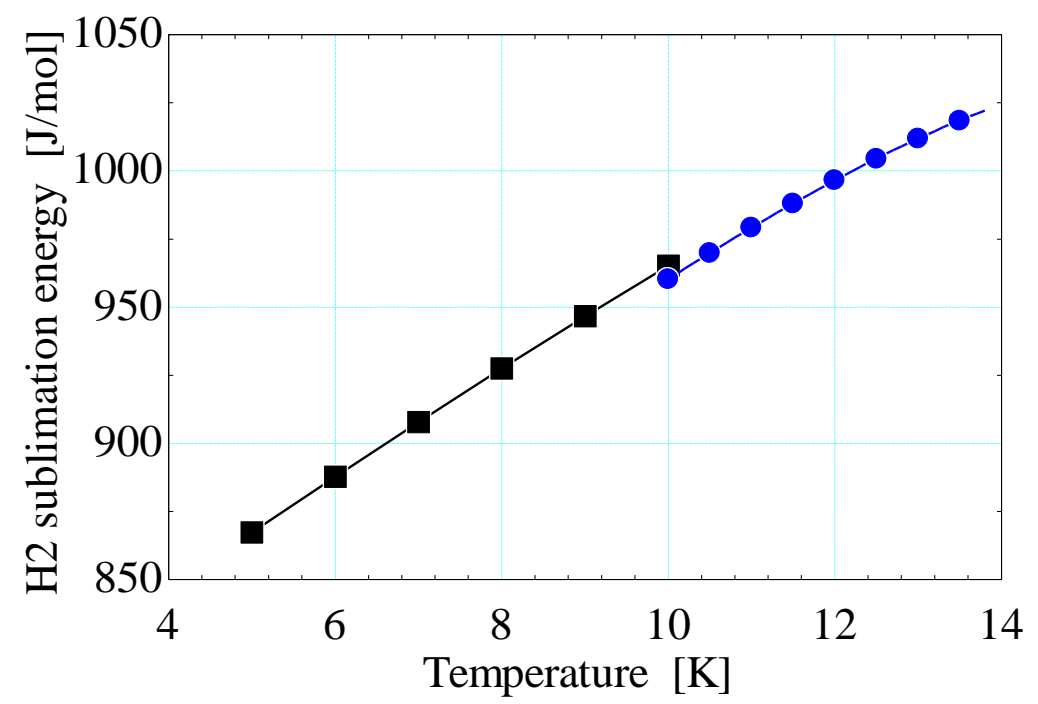

FIGURE 2. Hydrogen sublimation energy as a function of temperature

Various modeling efforts have been carried out for different types of diffusion problems [15-18]. These models are generally one-dimensional, rely on the existing mass diffusivities [19-24], at times do not include a momentum balance, and usually do not consider the temperature or pressure dependence of the thermal and kinetic properties. Mass diffusivity varies significantly in different conditions and is modeled for different driving forces. For example, the Chapmann-Enskog correlation models the isothermal and isobaric mass diffusivity [19]. Thermally driven mass diffusion alone is also available in the open literature [25]. Besides density and thermally driven diffusion, chemical potential driven diffusion has also been discussed [24]. The above numerical models are limited by their ability to solve complex coupled non-linear equations.

When the pump surface temperature is below the triple-point temperature of hydrogen, hydrogen molecules in the bulk flow begin to deposit onto the pump surface, and experience a phase change from vapor to solid. After the first five or so monolayers 
are deposited, the hydrogen molecules in the gas flow see and interact with the solid hydrogen molecules on the pump surface instead of the pump surface directly (or indirectly). This interaction is the main mechanism for the ITER cryoviscous compressor. In other words, deposition, the phase change of the hydrogen molecules from vapor to solid, is the main adsorption mechanism for the ITER cryoviscous compressor.

Deposition is a kinematic process. Molecules enter the solid phase from the vapor phase and leave the solid phase to the vapor phase at the same time. The equilibrium vapor pressure is the pressure at which the vapor phase and the solid phase reach equilibrium. Whenever the gas pressure adjacent to the solid is larger than the vapor pressure associated with the solid temperature, there will be a net transfer of molecules going from the gas into the solid phase. Alternately, whenever the gas pressure adjacent to a solid is smaller than the vapor pressure associated with the solid temperature, a net transfer of molecules from the solid into the gas phase occurs.

As shown in Fig. 3, the equilibrium vapor pressure of solid hydrogen is a strong function of temperature [13-14]. Because of its importance to the analysis that follows below, the data is also summarized in table 1 .

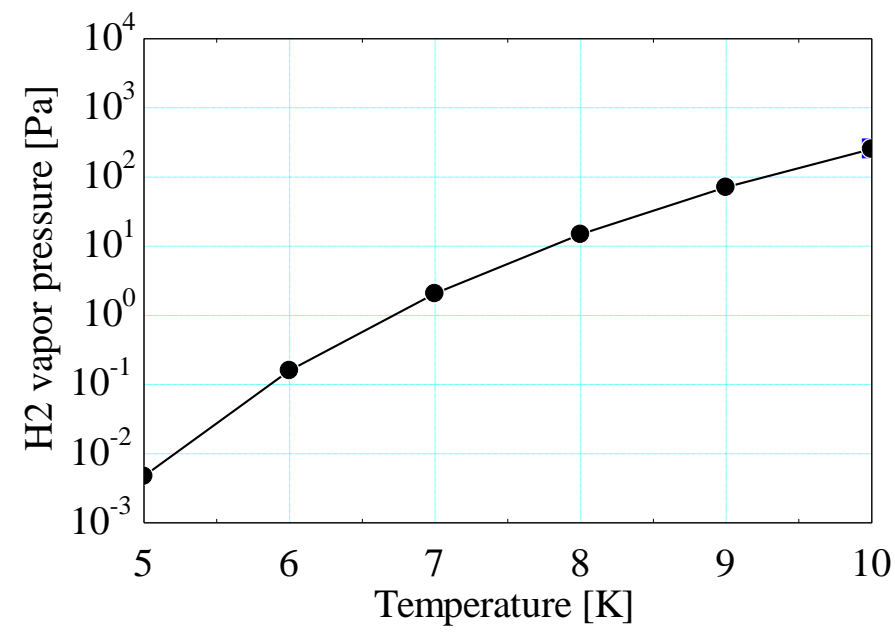

FIGURE 3. Hydrogen solid vapor pressure as a function of temperature [5 K, $10 \mathrm{~K}]$.

TABLE 1. Hydrogen vapor pressure as a function of temperature

\begin{tabular}{ccccccc}
\hline $\begin{array}{c}\text { Temperature } \\
{[\mathbf{K}]}\end{array}$ & 5 & 6 & 7 & 8 & 9 & 10 \\
$\begin{array}{c}\text { Vapor pressure } \\
{[\mathbf{P a}]}\end{array}$ & 0.00476 & 0.16 & 2.08 & 14.91 & 71.23 & 255.58 \\
\hline
\end{tabular}

In view of the above observations, one may conclude that the pump surface temperature is a key factor for pumping performance. The saturation pressure of hydrogen is very sensitive to temperature. Note that in the range of 5-8 K, a one-degree change in temperature produces an order-of-magnitude change in the hydrogen vapor pressure. Furthermore, in that the gas pressure determines a transition from viscous to molecular flow regimes, we may observe that the temperature of the CVC wall strongly impacts the flow regime. As mentioned above, as the wall temperature approaches $6 \mathrm{~K}$, 
the gas dynamics will transition between viscous and molecular flow regimes. The following analyses detail the impact that the mass, momentum and energy transfer have on the CVC wall temperature profile.

\subsection{Mass, momentum, and energy balance}

The ITER CVC presents unique features for transport phenomena. Because of the adsorption of hydrogen molecules on the pump surface and its sensitivity to the surface temperature, the transport of species, momentum, and energy in the control volume of the hydrogen gas flow are all significantly affected by the boundary condition defined by the surface. In the experimental data gathered at ORNL, this boundary condition varies with time and position, and is strongly influenced by the inlet helium coolant. The following model is constructed to directly address the experimental data gathered at ORNL.

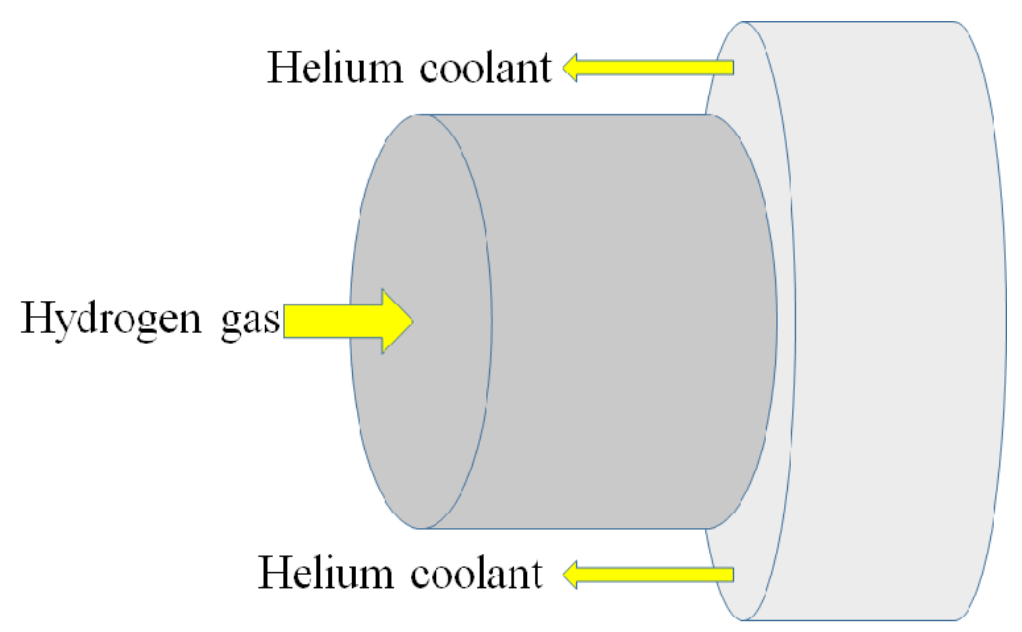

FIGURE 4. Flow configuration of the cryo viscous compressor.

As shown in FIGURE 4, the CVC sub-scale test rig is modeled as a concentric tube-in-tube heat exchanger. The hydrogen gas (or a hydrogen-helium gas mixture) flows through the inner tube, while the helium coolant flows through the annulus. The tube wall separating the two flows develops a temperature profile that is determined by the heat transfer between the two fluids, which in turn is influenced by accumulation of solid hydrogen on the wall. As hydrogen gas flows through the tube, heat is transferred from the bulk gas flow to the cryopump surface and then is taken away by the helium coolant. As a result, the temperature of the hydrogen flow decreases as it flows through the tube. Because of its relatively low pressure, a sufficient decrease in the temperature of the hydrogen flow results in the deposition of solid, rather than liquid hydrogen on the pump wall.

The inner surface of the CVC subscale test rig defines the physical and computational boundary for the hydrogen flow inside the inner tube. To be specific, the surface temperature sets the temperature boundary for the hydrogen flow, and the axial bulk velocity is zero at the pump surface.

In the experiment performed at ORNL, a mass flow controller determines the inlet mass flow rate of the hydrogen gas. The data show that this mass flow rate can be well 
maintained as a desired constant. In addition, the inlet hydrogen gas is precooled by a 77$\mathrm{K}$ liquid nitrogen bath, so that the inlet hydrogen gas temperature can be reasonably assumed constant at $77 \mathrm{~K}$.

As shown in the following equation, the mass flow rate $\dot{m}$ is the product of density $\rho$, bulk velocity $u$ and cross-sectional area $A$. Also, the hydrogen density is a function of pressure $P$ and temperature $T$. Thus, the inlet hydrogen flow velocity is determined from the inlet mass flow rate, temperature, and hydrogen pressure.

$$
\dot{m}=\rho(P, T) u A
$$

With a constant inlet mass flow rate, and in the absence of adsorption or sublimation, the upstream hydrogen pressure and inlet hydrogen velocity are constant. When adsorption or sublimation occurs, both of them change with time.

As the hydrogen gas flows through the inner tube, its bulk velocity decreases. There are three reasons for the decrease: friction, cooling, and adsorption of hydrogen molecules on the wall. The decrease in velocity due to friction is constrained by the conservation of momentum. The decrease in velocity is determined by the cooling effect, and adsorption impacts the velocity as a result of the conservation of species (mass).

The cooling power of the CVC is provided by the helium coolant, and determined by its inlet temperature, mass flow rate and flow path geometry.

As mentioned above, a one-degree change in the surface temperature of the pump wall yields orders of magnitude change in the hydrogen vapor pressure. Thus, to the degree that the inlet helium coolant temperature and mass flow rate influence the wall temperature of the cryopump, they influence its cooling power and overall operation. The pump geometry also influences the cooling power through its influence on the heat transfer coefficients associated with convection along the inner wall and in the annular region.

\subsection{Model and governing equations}

A transient numerical model has been constructed to accurately characterize the time-dependent changes of the variables observed in the experimental data. The experimental data present the test results for which the warm gas flow is pure hydrogen gas, and include time dependent information from five temperature measurements (two for the helium coolant and three for the pump wall surface) and one pressure measurement for the inlet hydrogen flow pressure (upstream hydrogen flow pressure).

The experimental data display mass flow rates for the hydrogen gas and the helium coolant that remain quite stable with time. Therefore, the transient model treats the mass flow rates of the hydrogen gas and the helium coolant as constants, and treats the inlet hydrogen gas pressure and inlet helium coolant temperature as input boundary conditions. Using these data as given information, the model then calculates the other variables (temperature, pressure, density, and velocity) at each spatial node for discrete time steps. The modeling results are then compared with the experimental data. 
3.3.1. Control Volume Analyses: The helium coolant velocity, about $0.3 \mathrm{~cm} / \mathrm{s}$, is orders of magnitude smaller than the hydrogen gas velocity. The numerical time step size for the model is set at about half of the time it takes for the helium coolant to travel between two adjacent nodes. As a result, the hydrogen gas is treated as a steady-state control volume, while the helium-coolant-plus-pump-wall is treated as a transient-state control volume. The justifications for this choice are as follows:

For the control volume of the hydrogen gas, the number of hydrogen molecules traveling through a numerical node in one time step is much more than the change in the number of molecules contained in the node. Considering the energy balance for the node in the form: IN = OUT + STORED, the energy represented by the IN or OUT terms is thus much larger than the energy represented by the change in the STORED term. In other words, the hydrogen gas gives energy away to the helium coolant by passing a large amount of flow through the node and decreasing the enthalpy of the flow, but not by decreasing the internal energy of the molecules stored in the node. As a result, the hydrogen gas is treated as a control volume at steady state.

For the control volume of the helium-coolant-plus-the-pump-wall, the amount of helium traveling through one numerical node in one time step is much smaller than that contained in the node. In the energy equation IN = OUT + STORED, the energy represented by the change in the STORED term is much larger than the energy associated with the IN or OUT terms. In other words, the helium coolant (plus the pump wall) primarily absorbs energy from the hydrogen gas by increasing the internal energy associated with the STORED term, but not by carrying it away with the enthalpy of the coolant passing through the node (the IN and OUT terms). As a result, the temperature at each node increases with time. The control volume of the helium-coolant-plus-the-pumpwall is thus treated as a transient control volume.

The transient model progresses in the following manner: Initial conditions are defined for the pump wall and the helium coolant temperatures, as well as the inlet conditions for the hydrogen gas and the helium coolant. The temperature, pressure, density, and velocity values for the hydrogen-gas control volume are then calculated for each axial position based on the inlet hydrogen gas condition and the pump wall temperature at that time. The heat flux from the hydrogen gas to the pump wall is also calculated. Next, for the control volume of the helium-coolant-plus-the-pump-wall, the heat flux from the hydrogen gas is used together with the inlet helium coolant condition to calculate the coolant properties at all the nodes for the next time step. Finally, the new pump wall temperature together with the inlet hydrogen gas condition is used to calculate the steady-state profiles for the hydrogen gas at the next time step.

The approach used by the transient model is depicted in FIGURE 5. Note the two separate control volumes for the steady-state hydrogen gas and the transient-state heliumcoolant-plus-the-pump-wall. 


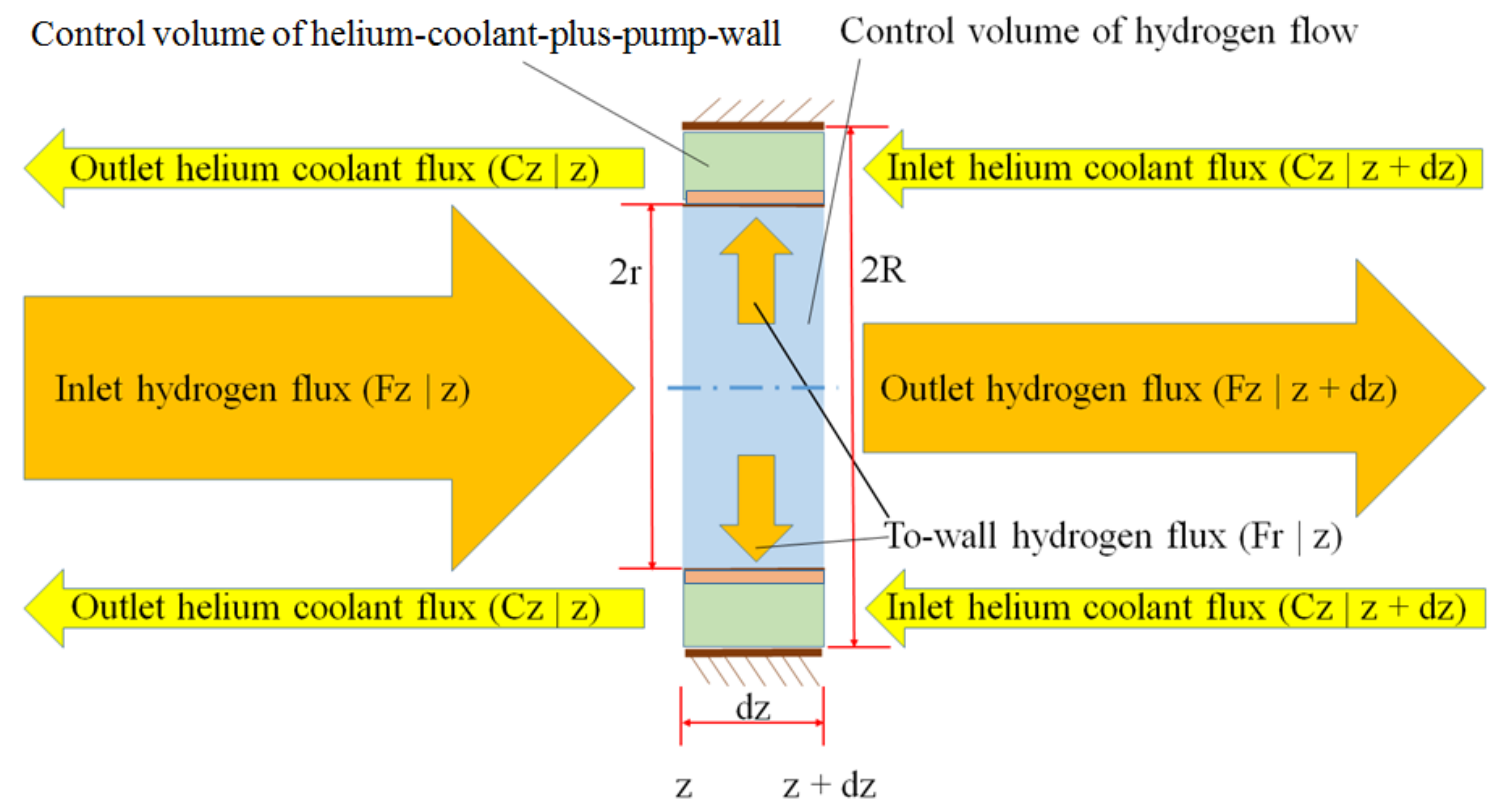

FIGURE 5. Control volume of the hydrogen flow at steady state and control volume of the helium-coolant-plus-pump-wall at transient state.

3.3.1.1 Hydrogen control volume: For the control volume of the steady-state hydrogen gas, the general governing equation for the flux in axial (z) direction is as follows:

$$
r \frac{d\left[F_{z}\right]}{d z}+2 F_{r}=0 .
$$

The general equation can be expressed to represent the conservation in species, $\mathrm{z}$ momentum, and energy respectively.

$$
\begin{gathered}
r \frac{d\left[M_{z, H_{2}}\right]}{d z}+2 M_{r, H_{2}}=0 . \\
r \frac{d[z z]}{d z}+2 \quad r z=0 . \\
r \frac{d\left[E_{z}\right]}{d z}+2 E_{r}=0 .
\end{gathered}
$$


In the conservation equation of the hydrogen species, the mass flux terms are developed as follows to represent the hydrogen mass flux in the axial (z) direction and in radial (r) direction respectively.

$$
\begin{gathered}
M_{z, H_{2}}={ }_{H_{2}} u_{z} \\
M_{r, H_{2}}=h_{m}\left(\begin{array}{ll}
H_{2} & H_{2}, w
\end{array}\right)
\end{gathered}
$$

The mass transfer coefficient $h_{m}$ is developed in the similar manner as the heat transfer coefficient $h_{t}$, and the two transfer coefficients are calculated as follows.

$$
h_{t}=\frac{N u \times k}{2 r}, h_{m}=\frac{S h \searrow_{a b}}{2 r}
$$

The Nusselt and Sherwood numbers are obtained from built-in functions within the Engineering Equation Solver (EES) software package [26] for internal pipe flow, and based on corresponding correlations as defined in Nellis \& Klein [27]. The lower limiting case is assumed for the heat and mass transfer coefficients, based on a constant wall temperature.

In the conservation equation of $\mathrm{z}$-momentum, the z-momentum flux terms are developed as follows to represent the $\mathrm{z}$-momentum transfer in axial ( $\mathrm{z}$ ) direction and in radial (r) direction respectively.

$$
\begin{gathered}
{ }_{z z}{ }_{H_{2}} u_{z} u_{z}+P \\
=P_{\text {friction }}+M_{r, H_{2}} u_{z}
\end{gathered}
$$

where the pressure loss due to friction $\Delta P_{\text {friction }}$ is related to the friction factor $f$ in the usual way:

$$
P_{\text {friction }}={ }_{H_{2}} f \frac{u_{z}^{2}}{2} \frac{d z}{2 r}
$$

In the conservation of energy equation, the energy flux terms are developed as follows to represent the energy flux in axial ( $\mathrm{z}$ ) direction and in radial (r) direction respectively. 


$$
\begin{gathered}
E_{z}={ }_{H_{2}} u_{z} h_{H_{2}}+{ }_{H_{2}} u_{z} \frac{u_{z}^{2}}{2} \\
E_{r}=M_{r, H_{2}}\left(h_{H_{2}}+\frac{1}{2} u_{z}^{2}\right)+h_{t}\left(\begin{array}{ll}
T & T_{w}
\end{array}\right)
\end{gathered}
$$

In addition to the conservation equations, the ideal gas equation of state is used because of the close-to-unity compressibility factor.

$$
P=R_{m} T
$$

3.3.1.2 Helium coolant control volume: A lumped parameter approach is used for the control volume of the transient-state helium-coolant-plus-pump-wall (abbreviated as helium coolant), and axial conduction is neglected. As justification for this approach, we note that the Biot number, defined in the usual way as:

$$
B i=\frac{t h_{w} h_{\text {coolant }}}{k_{w}}
$$

is typically less than 0.1 for all conditions encountered in the model.

The control volume of the helium coolant is coupled to the control volume of hydrogen gas by the radial energy flux. The radial energy flux from the hydrogen gas to the cryogenic pump wall $E_{r, H 2} \rightarrow^{w}$ is the sum of the energy change associated with the radial hydrogen flux and the convection heat transfer flux.

$$
E_{r, H_{2}>w}=M_{r, H_{2}}\left(h_{H_{2}}+\frac{1}{2} u_{z}^{2}\right)+h_{t}\left(\begin{array}{ll}
T & T_{w}
\end{array}\right)
$$

The enthalpy change of the hydrogen gas $\Delta h_{H 2}$ at a given axial node is the sum of the enthalpy change from the gas phase in the bulk flow to the gas phase at the gas-solid equilibrium condition at the wall, and the enthalpy change from the gas phase in the gassolid equilibrium condition to the corresponding solid phase.

$$
h_{H_{2}}=h_{H_{2}, \text { gas }>\text { gas }}+h_{H_{2}, \text { gas }}>\text { solid }
$$


The energy equation governing the control volume of helium-coolant-plus-pumpwall is given by

$$
E_{r, H_{2}>w} A_{s}=Q_{r, w>\text { coolant }}+\frac{U_{w}}{t}
$$

where the term $\Delta U_{w} / \Delta t$ represents the rate of change of the pump-wall internal energy. The Energy transfer rate from the pump wall to the helium coolant $\left(Q_{r, w} \rightarrow\right.$ coolant $)$ is developed as the following equation.

$$
Q_{r, w>\text { coolant }}=H_{\text {coolant }}+\frac{U_{\text {coolant }}}{t}
$$

where the term $\Delta U_{\text {coolant }} / \Delta t$ represents the rate of change of the helium-coolant internal energy.

Additionally, $Q_{r, w} \rightarrow^{\text {coolant }}$ can be expressed by the following equation.

$$
Q_{r, w>\text { coolant }}=\frac{T_{w} T_{\text {coolant }}}{R_{\text {coolant }}}
$$

where the term $R_{\text {collant }}$ represents the thermal resistance between the pump wall and the helium coolant flow. The thermal resistance is dominated by the convective heat transfer that ranges between 30 and $100 \mathrm{~W} / \mathrm{m}^{2} \mathrm{~K}$.

It takes about 377 seconds for the helium coolant to travel though the pump. The history of the helium coolant thus affects the initial temperature of the helium coolant contained in the pump at the beginning of the time, a feature that is included in the model.

The initial pump-surface temperature, although not directly measured at all of the model nodes, is estimated by a linear interpolation of the experimental data. 


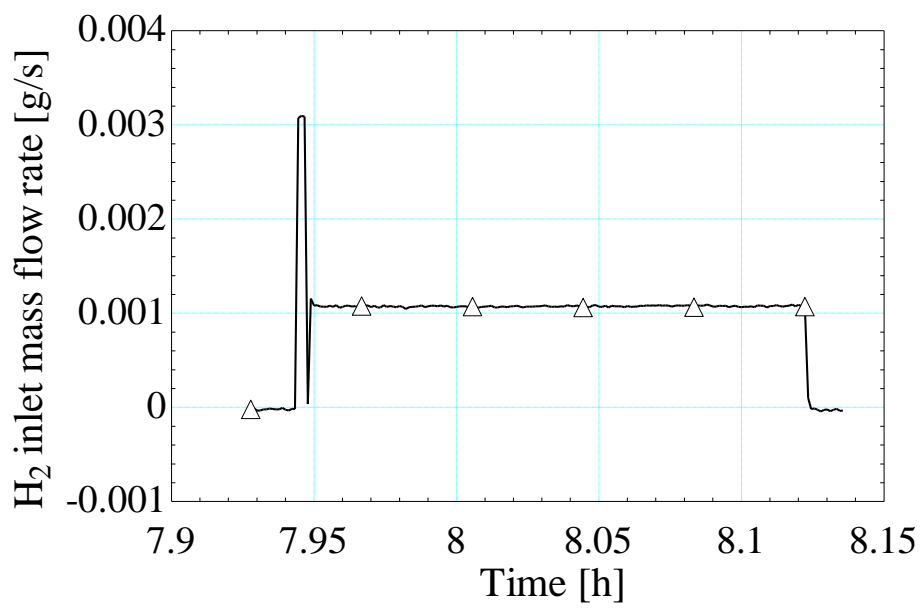

FIGURE 6. Hydrogen inlet mass flow rate as a function of time.

3.3.4. Input data for the model: Figure 6 shows the inlet hydrogen mass flow rate as controlled in the experiment. After the hydrogen mass controller is turned on, the mass flow rate initially rises to about $0.003 \mathrm{~g} / \mathrm{s}$ for the first 12 seconds, falls to near $0 \mathrm{~g} / \mathrm{s}$ for the next 6 seconds, and finally remains constant at about $0.0011 \mathrm{~g} / \mathrm{s}$.

In order to compare with the experimental data, the initial time of the transient model is set at the 7.95-hour mark as recorded by the experiment.

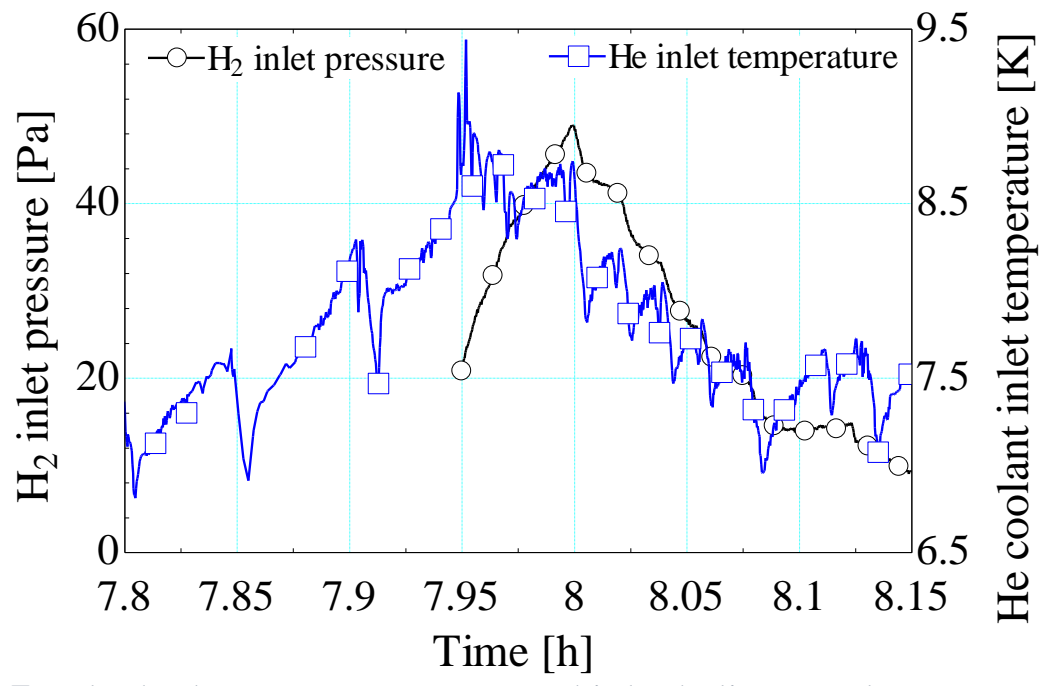

FIGURE 7. Inlet hydrogen gas pressure and inlet helium coolant temperature as a function of time.

Figure 7 shows the measured time dependence of the inlet hydrogen gas pressure and the inlet helium coolant temperature. Note, as mentioned above, that the accuracy of the pressure data is $0.5 \%$ of the measurement, while that for the temperature is $+/-0.03 \mathrm{~K}$. The inlet hydrogen pressure follows the trend of the inlet helium coolant temperature in a delayed manner. These two measurements are used as input parameters for the transient 
model. In the experiment, the data are collected every second. However, since the time step in the transient model is about 3.7 seconds, the experimental data is averaged for every 4 seconds.

\subsection{The Group-Member numerical technique}

A novel numerical technique, here named the Group-Member numerical technique, is used to solve the previously defined set of equations with highly coupled non-linear variables. The Group-Member numerical technique combines an explicit numerical technique with a non-linear equation solving technique, and is basically comprised of three steps as shown in Figure 8. The three steps are Grouping, Numerically Forward Marching, and Non-linear Equation Solving. In the Group-Member numerical technique, the individual unknown variables, which are the ultimate targets to be solved, are referred to as Members, and the combinations of those individual variables, which are the intermediary targets to be determined, are referred to as Groups. In fact, the technique might also have been named the Member-Group-Member numerical technique according to its procedure.

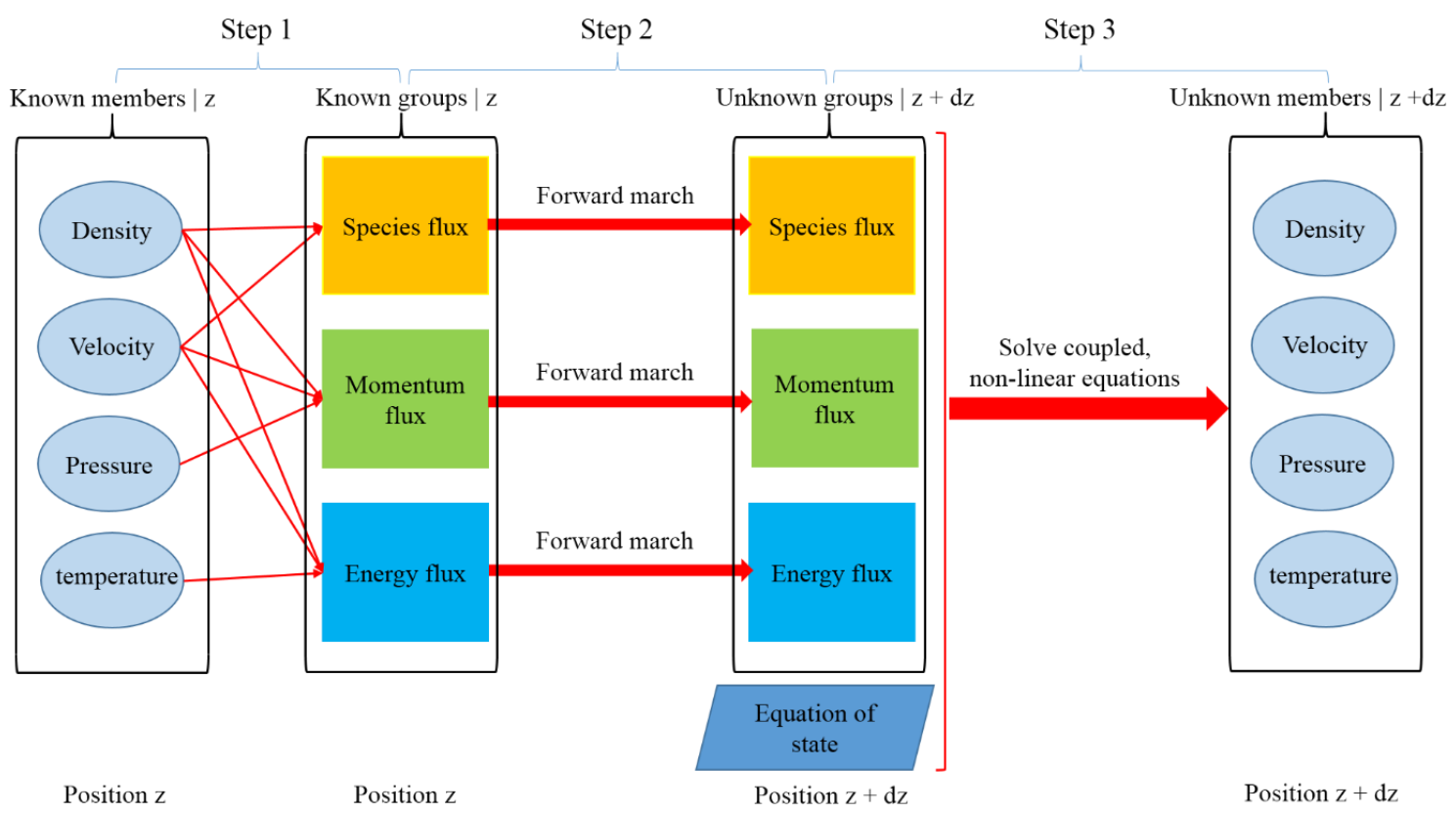

FIGURE 8. A flowchart of Group-Member numerical technique for the 1-D numerical model.

In the first step of the Group-Member technique, the individual ultimate target variables (Members) are grouped into several combinations (Groups) that have physical significance and enable the numerical solution to easily march forward. In this particular case, the Members are hydrogen density, helium density, axial bulk velocity, bulk temperature, hydrogen pressure, and helium pressure. The Groups are hydrogen mass flux, helium mass flux, axial momentum flux, and energy flux. Since the information of 
all the Members at the calculation boundary are known, the information of the Groups at the calculation boundary are also known. In the particular case, the inlet densities, bulk velocity, bulk temperature, and pressures are known, and thus the inlet mass fluxes, momentum flux, and energy flux values are known. There are no assumptions or approximations in step one, and therefore there are also no numerical errors in step one. Step 1 packages the information in preparation for Step Two. It should be noticed that the six Members are combined into four Groups, which is equal to the number of the governing equations. In other words, the number of the Groups is not determined by the number of Members but is determined by the number of governing relationships that would be used for numerically marching forward in Step Two. No information is missing as a result of the grouping because the two equations of state (of hydrogen and helium) are directly adopted in Step Three, and there is no need to numerically march forward using the two equations of state.

Step two, numerically marching forward, explicitly determines the new Group values at a new position under the constraint of the governing equations. The governing equations used in Step Two are defined by the physics governing the process of a problem, and thus control the differential change of the variables (Groups). In this particular case, the governing equations are the balance equations of species, momentum, and energy, and they constrain the differential change of mass flux, momentum flux, and energy flux. The explicit numerical method adopted in Step Two determines the order of the Group-Member numerical technique. In this particular case, Euler's method is adopted as the explicit numerical method, and thus the Group-Member numerical technique is first order. If higher order methods are adopted, the order of the GroupMember numerical technique will be increased accordingly. One difference from the common numerical method is that in Step Two, only one numerical step is taken, which means that Groups are only calculated at the position of $z+d z$ based on the values at the position of $z$, and then Step Two is over. The result of Step Two is that the Groups at the new position of $\mathrm{z}+\mathrm{dz}$ are now known values. Furthermore, the combinations of the ultimate individual variables (Members) are known values as well.

Step three, solving the non-linear equations, solves for the ultimate individual variables (Members) at the new position of $\mathrm{z}+\mathrm{dz}$ based on the value of the Groups at the new position of $z+d z$, which are numerically solved in Step Two. The values of the four Groups generate four equations, which, along with the additional equation of state for hydrogen and helium provide enough equations to solve for the individual Members. As long as the coupled non-linear equations are based on appropriate physics, a proper method should be able to find them.

Using the Group-Member numerical technique, the one-dimensional numerical model is solved using the Engineering Equation Solver software, or EES.

The innovation in the Group-Member numerical method is to connect the two separate domains, namely the numerical method and the equation solving technique. The Group-Member numerical technique takes advantage of both the numerical technique and the non-linear equation solving technique while at the same time avoiding the cost of both methods. The explicit numerical technique is convenient for marching forward and generating a solution from a known starting point (an information source). However, in the existing situation of non-linear coupled variables it is usually necessary to linearize 
and uncouple those variables before numerically marching forward. The required effort and skill for the linearization process can be significantly increased with an increase in the complexity of equations. Furthermore, when equation parameters, such as conductivity and viscosity, are functions of unknown variables (temperature, pressure and composition) and cannot be treated as constants, it is even more time-and-effort consuming to solve those set of equations. Further, when the unknown variables appear inside a complicated operation, for example an operation involving the third power of the unknown variable, the linearization step could be impossible. On the other hand, a nonlinear equation solving technique is convenient for solving non-linear coupled equations. However, non-linear equation solving techniques can only work on a control volume at one position where the equations and unknowns are located. There is no information transfer from other positions. The Group-Member numerical technique takes advantage of both techniques.

The goal of the Group-Member numerical technique is to let information travel without tedious effort.

\section{Results and discussion}

The transient model uses 51 spatial nodes and 310 time steps to produce results for comparison with those reported by Oak Ridge National Laboratory [5,6]. The associated node length is $0.02092 \mathrm{~m}$ and the time step is 2 seconds.

Figures 9 to 19 present the results of the model. These are grouped according to the following three features: 1) time-dependent temperature profiles of the wall and coolant, 2) properties of the hydrogen gas flow, and 3) heat and mass transfer. The first set of results is directly compared with associated experimental measurements, demonstrates a reasonable agreement, and provides a solid confidence level for the remaining predictions of the model, especially those regarding heat and mass transfer as well as the hydrogen capture rate and location in the pump.

\subsection{Time dependent temperature profiles}

Because the wall temperatures are influenced by both the heat transfer from the warm hydrogen gas entering from one end, and the cold helium coolant from the other end, we display the time- and spatially- varying hydrogen gas temperatures before focusing on the wall temperatures. Figure 9 displays the calculated hydrogen gas temperature through the pump at four distinct times. The first time corresponds with the introduction of the hydrogen gas, while the other three are spaced over the same duration covered in Figure 6.

The dominant feature is a rapid decline in temperature over the first third of the pump length, followed by a slower but continual decline over the remaining length of the pump. Secondarily one can observe smaller time dependent changes at all locations. 


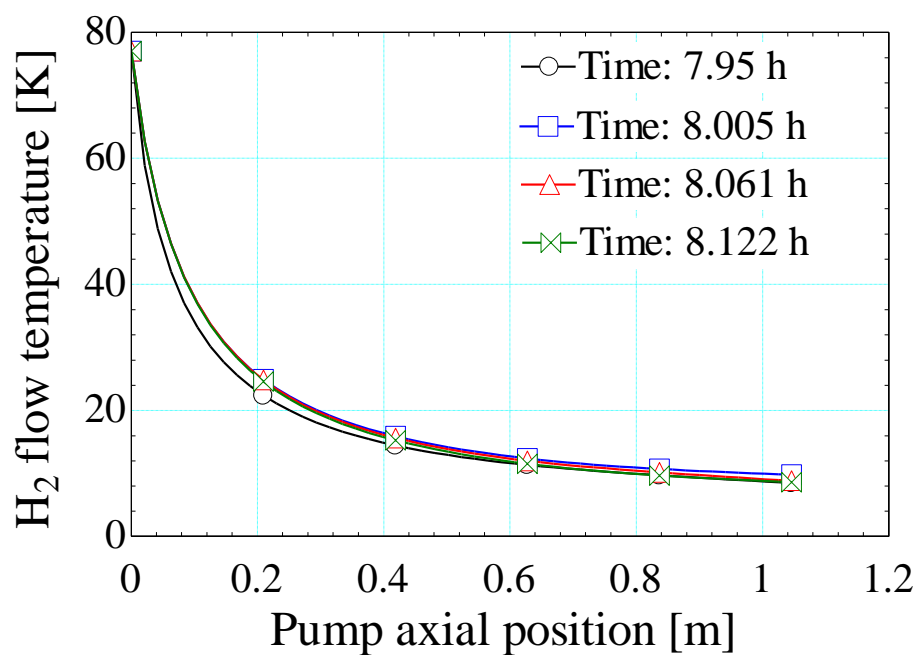

FIGURE 9. Hydrogen gas temperature as a function of pump axial position at 4 time steps.

Figures 10a-d display the direct comparison of the wall temperatures at the four locations reported from the experimental data while Figures 11 and 12 display the calculated overall spatial variation of the pump wall temperatures.

The helium coolant inlet temperature measurement shown in Figure 7, with all its fluctuations, is used as input for the numerical model, whereas the wall temperature measurements (Experimental data) displayed in Figures 10a-d reflect much less fluctuations, presumably due to a thermal capacitance associated with the thermometer attachment to the wall. The additional thermal capacitance artifact was not included in the model. The fluctuations present in the model results displayed in Figures 10a-d therefore reflect a faster time response than the wall temperature measurements, and one that is in keeping with the response of the thermometer that is in direct contact with the helium coolant.

The measured and calculated temperatures at location $T_{\text {wall,2, }}$, near the helium coolant inlet agree very well. As the location moves toward the other end of the pump, the agreement diverges somewhat, indicating the possibility of an inaccurate thermal resistance in the model between the two fluids, magnified by the larger heat transfer at that end. It is also possible that the thermometers, being mounted on the coolant side of the pump wall and imperfectly insulated from the coolant, measure a temperature intermediate to the wall and coolant. Regardless of this discrepancy, the model results especially in Figures 10a and 10b reflect the dominant influence that variations of the incoming helium coolant temperature has on the pump wall, and therefore as is shown below, on the pumping performance. 


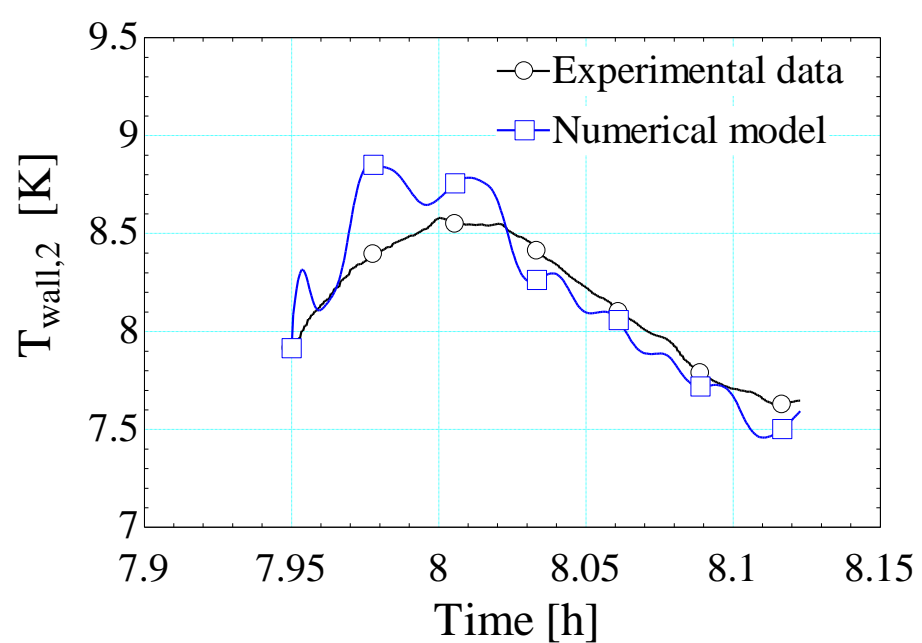

FIGURE 10a. Modeling result and experiment measurement for $T_{\text {wall, }, 2}$ as a function of time.

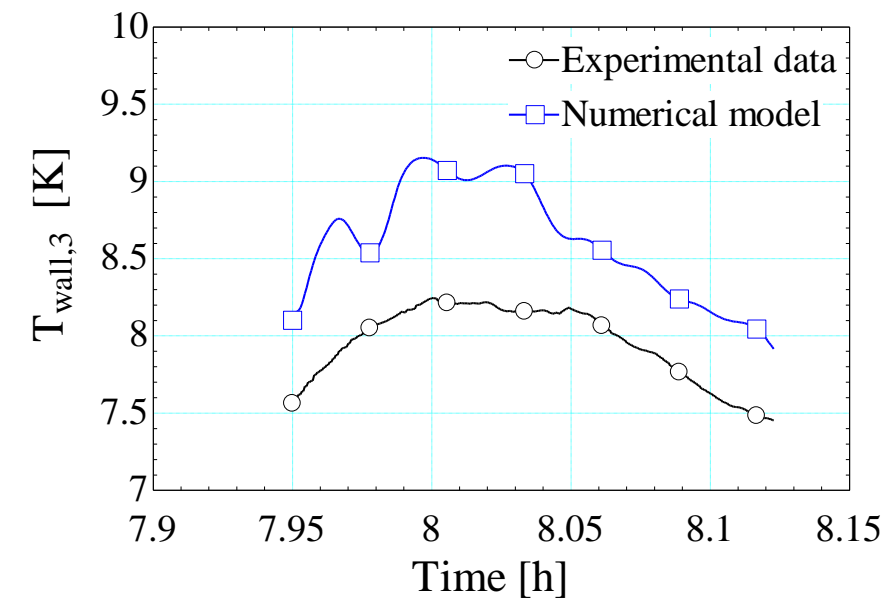

FIGURE 10b. Modeling result and experiment data for $T_{\text {wall }, 3}$ as a function of time.

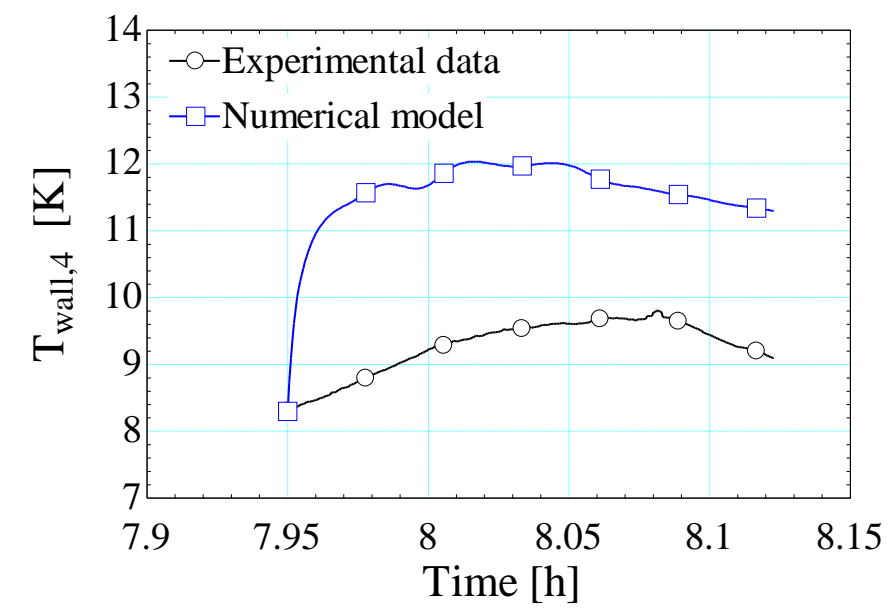

FIGURE 10c. Modeling result and experiment data for $T_{\text {wall, } 4}$ as a function of time. 


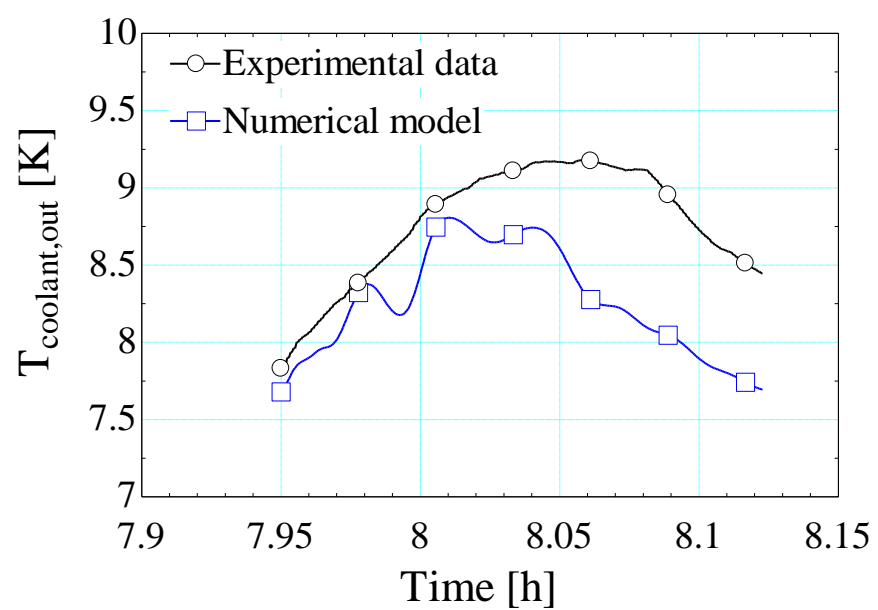

FIGURE 10d. Modeling result and experiment data for $T_{\text {outlet,He }}$ as a function of time.

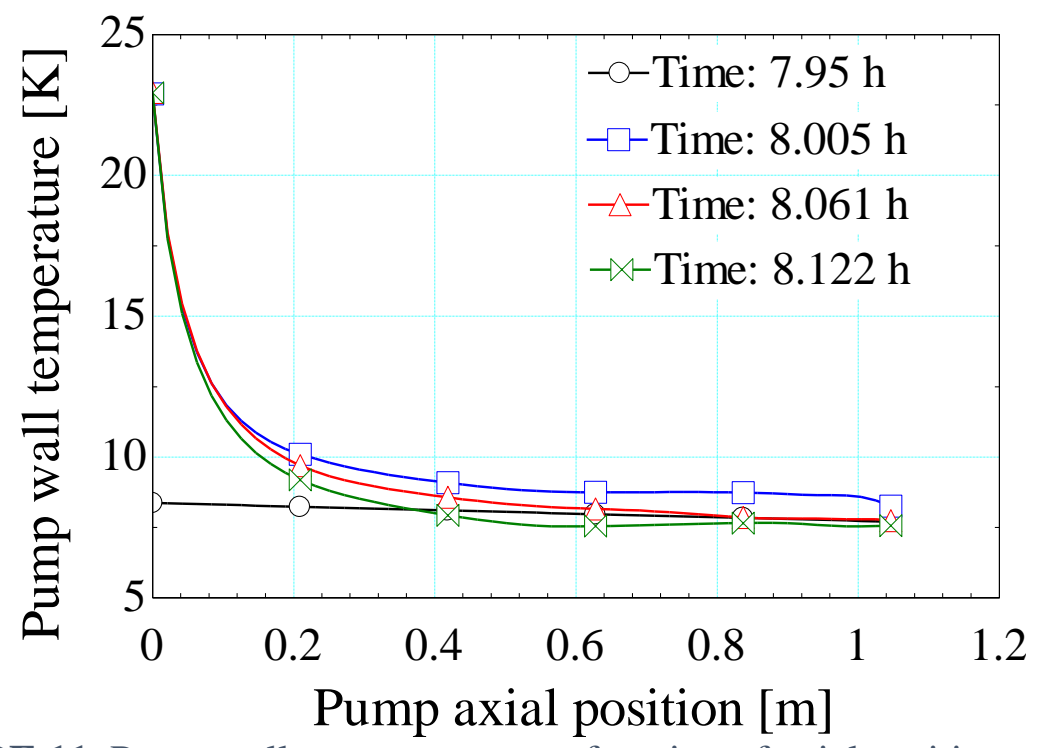

FIGURE 11. Pump wall temperature as a function of axial position at 4 time steps (I). 


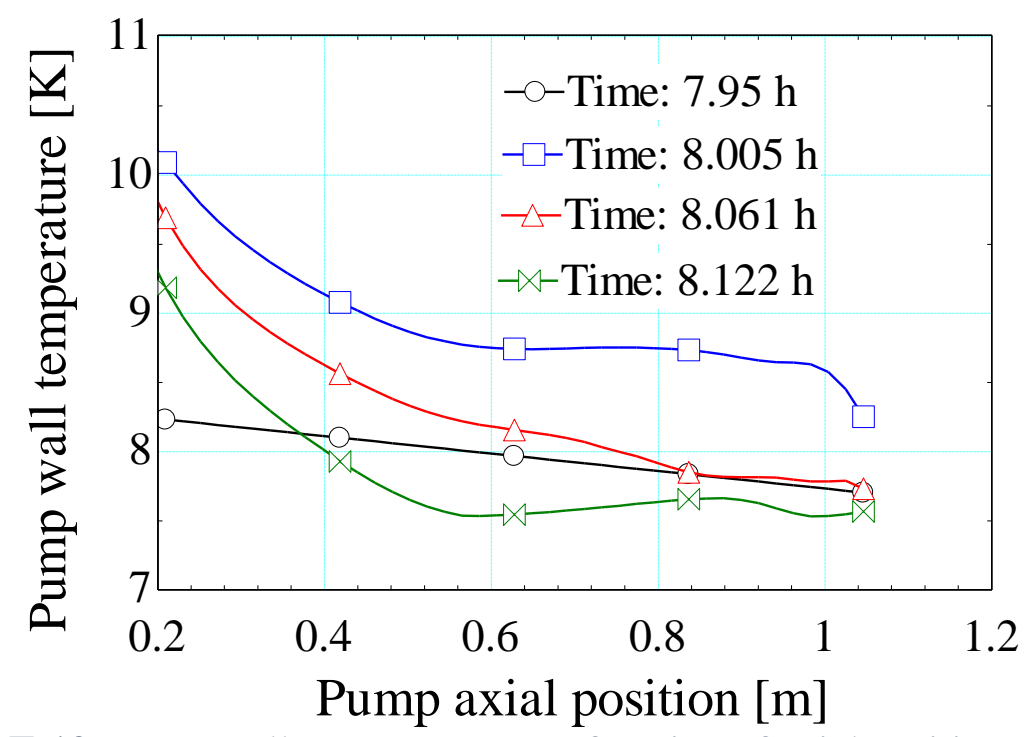

FIGURE 12. Pump wall temperature as a function of axial position at 4 time steps (II).

Several features of the above data are worth noting:

- In the experiment, the helium coolant precools the pump. The transient model thus sets the initial pump wall temperature (at Time: $7.95 \mathrm{~h}$ ) accordingly. Once the hydrogen gas is introduced, the pump wall temperature rises immediately at the pump entrance. The pump wall temperature at the hydrogen inlet end (near the axial position of $0 \mathrm{~m}$ ) is dominantly determined by the inlet hydrogen gas. Since the inlet hydrogen temperature is maintained as $77 \mathrm{~K}$, the pump wall temperature at the entrance is almost a constant.

- A large temperature drop of the gas occurs after it enters the pump. This feature is due to the large temperature difference between the hydrogen gas and helium coolant and the high heat transfer coefficient in this region. The hydrogen temperature drops to half of its inlet value within the first $0.1 \mathrm{~m}$. At the other end of pump, the hydrogen gas temperature is close to and dominantly determined by the inlet helium coolant. Note that it follows the same time dependence displayed in Figure 7.

- The temperature of the pump wall reflects a similar spatial and time dependence as the hydrogen gas. At the hydrogen gas-inlet end its temperature remains relatively constant, while at the helium coolant end the wall temperature follows the time dependence of the helium coolant. Recall from Table 1 that as the wall temperature drops from $9 \mathrm{~K}$ to $8 \mathrm{~K}$, the corresponding saturation pressure decreases from $80 \mathrm{~Pa}$ to $15 \mathrm{~Pa}$. Beyond the axial position of $0.2 \mathrm{~m}$, the wall is sufficiently cold for hydrogen molecules to be absorbed. Since the pump wall temperature is lower at the outlet end, the adsorption of hydrogen molecules occurs at the locations farther into the pump instead of near the entrance. This is a desirable feature ensuring that the pump entrance will not be blocked. 


\subsection{Features of the hydrogen gas flow}

In keeping with the time dependent measurement of the hydrogen pressure at its inlet shown in Figure 7, Figure 13 displays an initial increase and subsequent decrease of the hydrogen pressure. Additionally, the hydrogen pressure all along the pump length is shown, and due to the relatively small momentum loss at the wall, the pressure is spatially quite uniform.

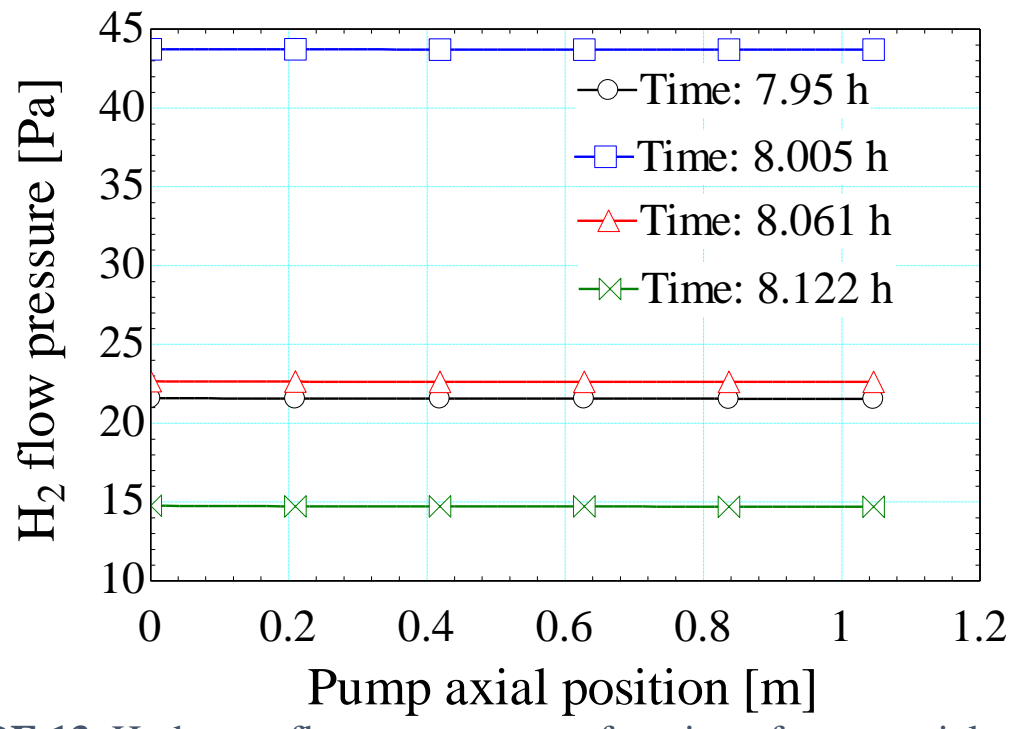

FIGURE 13. Hydrogen flow pressure as a function of pump axial position.

The variation of pressure with time is due to the wall-temperature-dependent sublimation or adsorption of the hydrogen molecules. The sublimation or adsorption process is a boundary condition for the control volume of the hydrogen gas. Any change in the boundary condition immediately affects the hydrogen gas pressure, because that information travels at the speed of sound. The sublimation or adsorption process depends strongly on the pump wall temperature.

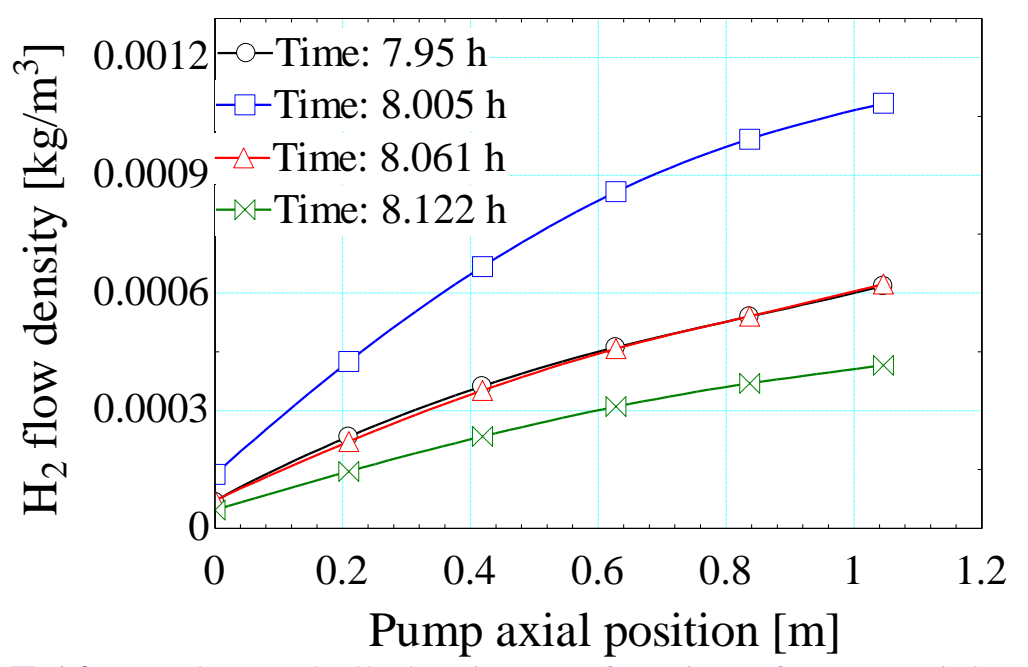

FIGURE 14a. Hydrogen bulk density as a function of pump axial position. 
Figures $14 \mathrm{a}$ and $14 \mathrm{~b}$ display the variation of hydrogen gas density as a function of time and location. The density goes up as the hydrogen gas travels into the pump, because the pump cools the gas. The inlet density variation with time reflects the time dependence of the inlet hydrogen pressure.

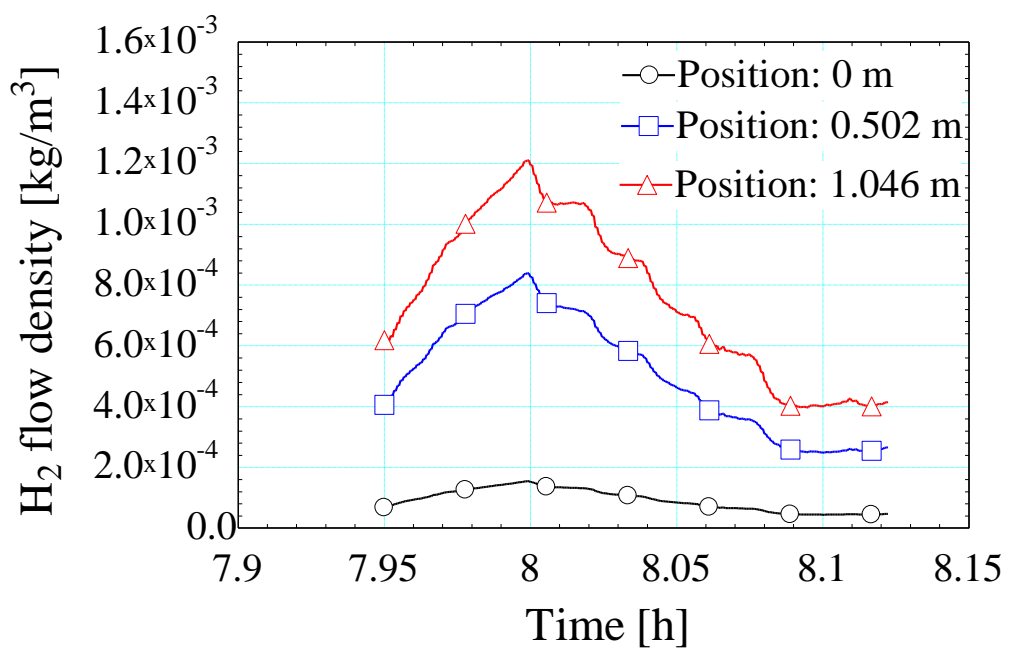

FIGURE 14b. Hydrogen bulk density as a function of time.

Figures $15 \mathrm{a}$ and $15 \mathrm{~b}$ display the variation of the axial hydrogen bulk velocity as a function of time and axial location. The axial hydrogen bulk velocity decreases as the gas travels into the pump, primarily as a result of cooling. As the gas is cooled and the pressure remains constant, the density rises, even though some mass is adsorbed at the wall. Conservation of mass results in a lower velocity as the density increases.

The inlet velocity is not a constant as a result of the changing inlet hydrogen pressure. The inlet hydrogen mass flow is maintained at a constant rate by the mass controller. The inlet temperature is maintained at a constant value by the nitrogen bath. The inlet pressure changes with time because of a changing rate of adsorption power due to the variable inlet helium coolant temperature. Because the inlet density varies according to the equation of state, the inlet velocity correspondingly varies in order to maintain a constant inlet mass flow rate.

The density increases to the maximum at the pump outlet while the bulk velocity decreases to the minimum at the same location. 


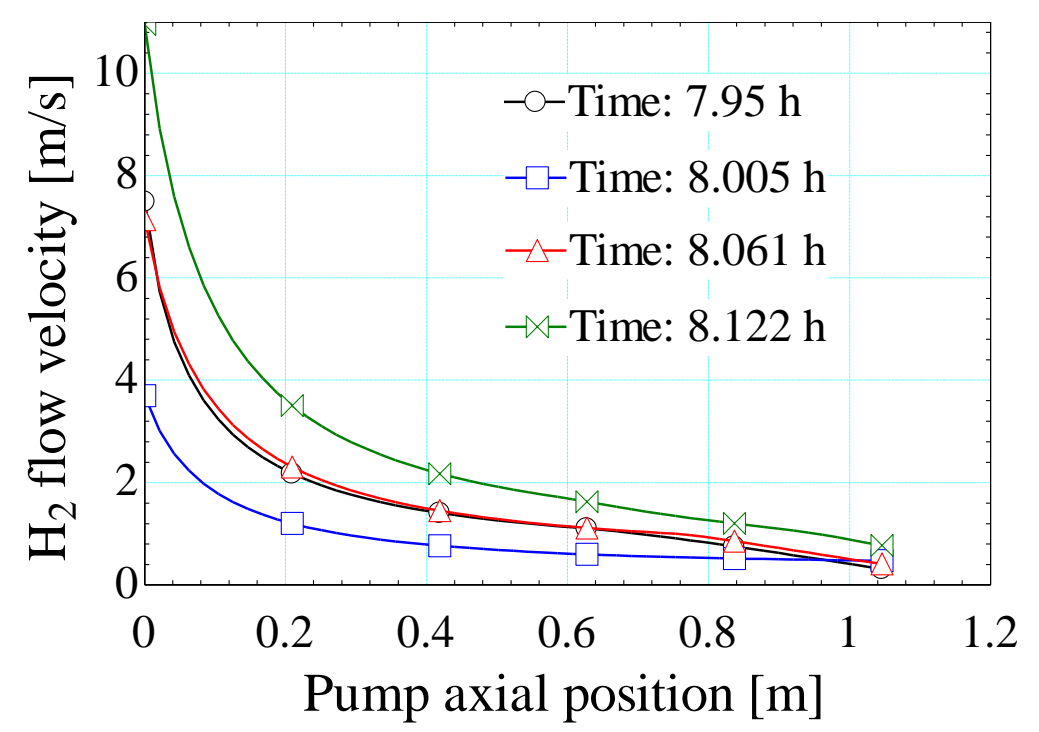

FIGURE 15a. Axial hydrogen bulk velocity as a function of pump axial position.

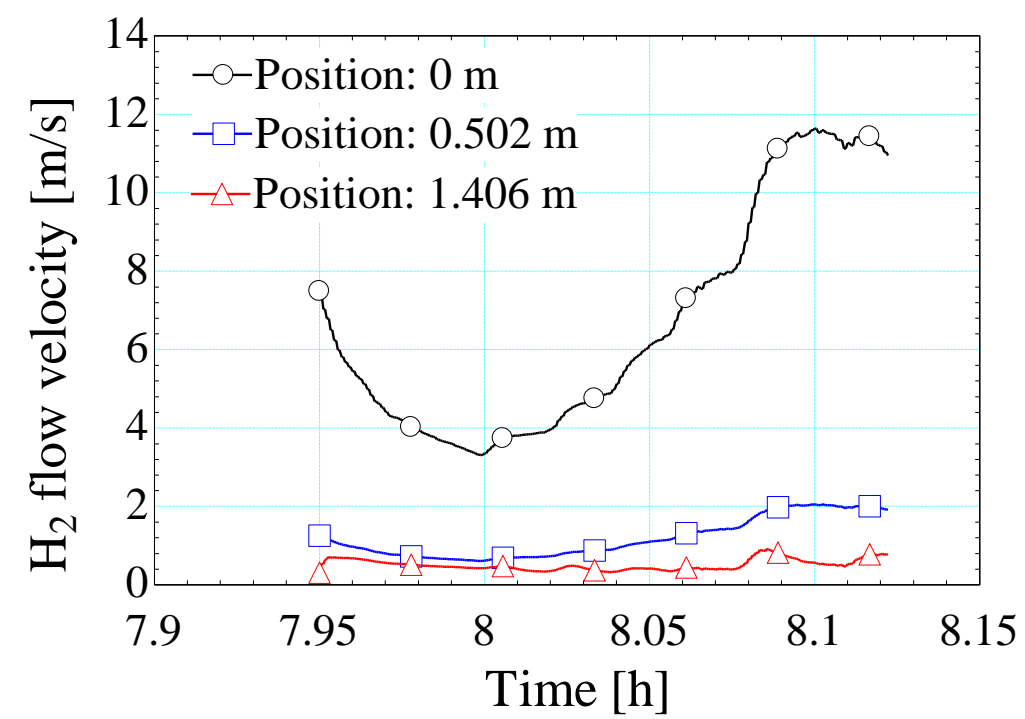

FIGURE 15b. Hydrogen bulk velocity as a function of time.

\subsection{Mass and heat transfer}

Figures $16 \mathrm{a}$ and $16 \mathrm{~b}$ present the radial mass flux of hydrogen to the wall as a function of time and position. The mass flux is the maximum at the Time of $7.95 \mathrm{~h}$ because the pump wall is precooled and initially at its lowest temperature. As the pump wall temperature increases, the mass flux decreases. The hydrogen molecules are primarily adsorbed in the downstream half of the pump, where the pump wall is cooled to sufficiently low temperatures so that the corresponding saturation pressure is less than the adjacent bulk hydrogen pressure. The deposition is modeled as the diffusion process driven by the hydrogen density difference. The model assumes that all hydrogen molecules that reach the wall remain there. 


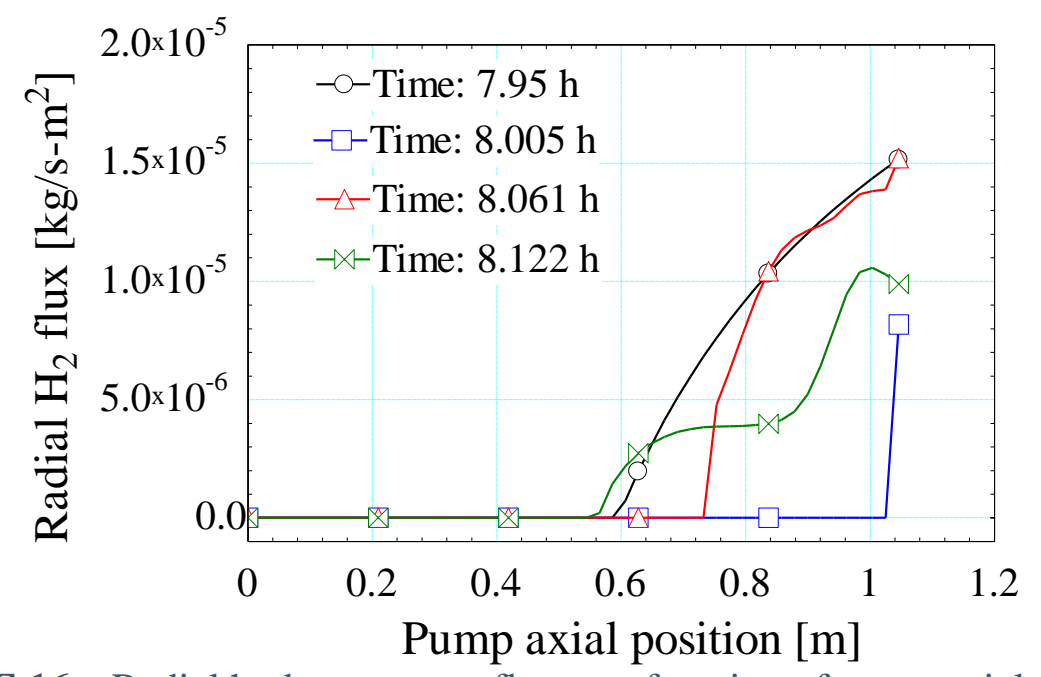

FIGURE 16a. Radial hydrogen mass flux as a function of pump axial position.

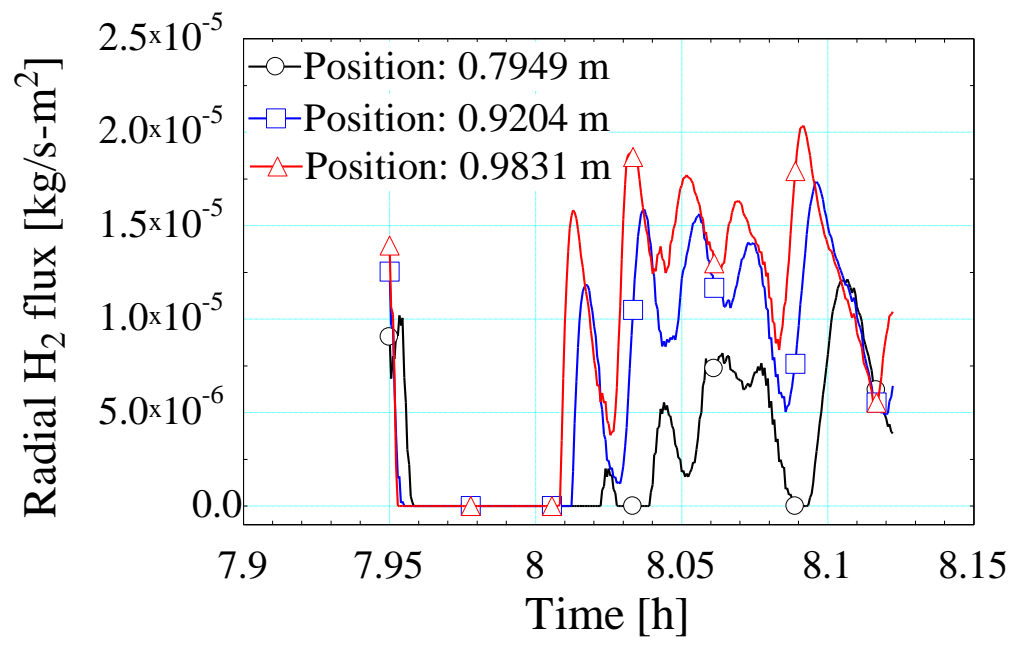

FIGURE 16b. Radial hydrogen mass flux as a function of time.

Figures $17 \mathrm{a}$ through $17 \mathrm{~b}$ display the variation of the radial energy flux as a function of time and location. The radial energy flux is the total energy flux transferred from the hydrogen gas to the pump wall, and is the sum of the convection heat transfer flux and the adsorption energy flux.

Most of the energy transfer occurs within the first $0.2 \mathrm{~m}$ because both the heat transfer coefficient and the temperature difference are high at the pump entrance. The profile remains nearly constant with time. 


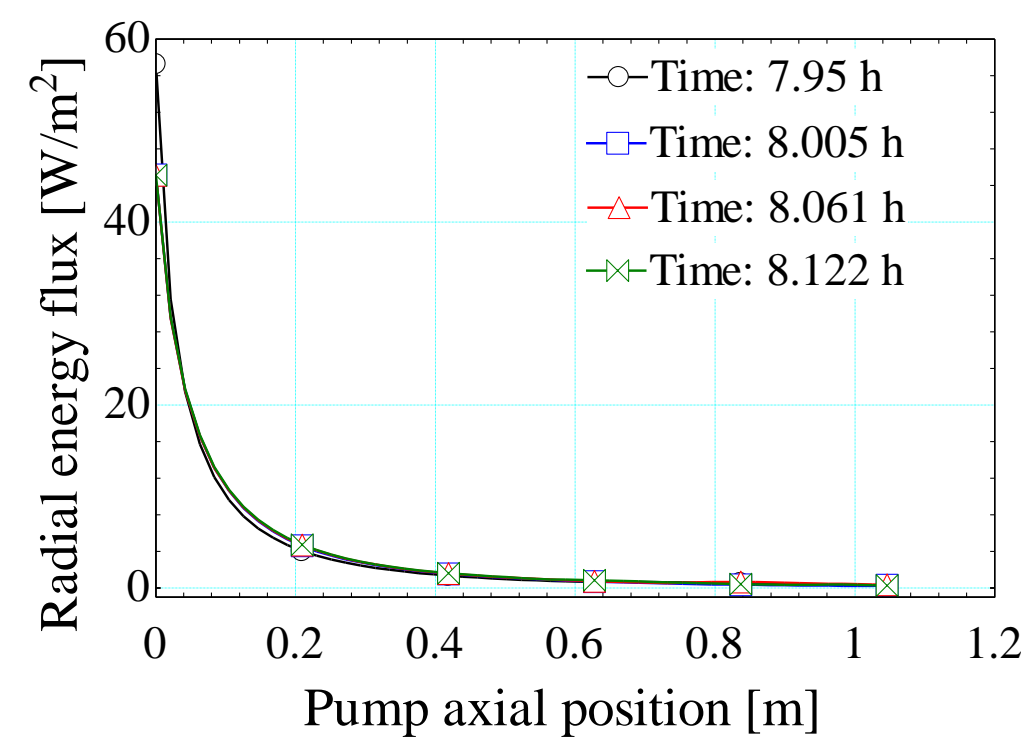

FIGURE 17a. Radial energy flux as a function of pump axial position.

Figure $17 \mathrm{~b}$ shows the profiles of the radial energy flux for only the second half of the pump, and provides a magnified view in the region where the contribution from adsorption is significant. The lumps in the profiles are the result of the adsorption heat of the hydrogen molecules. Note that the convective heat transfer is much larger than the adsorption heat. Figure 17c displays the same information as in Figure 17b, but at three specific locations and as a continual function of time.

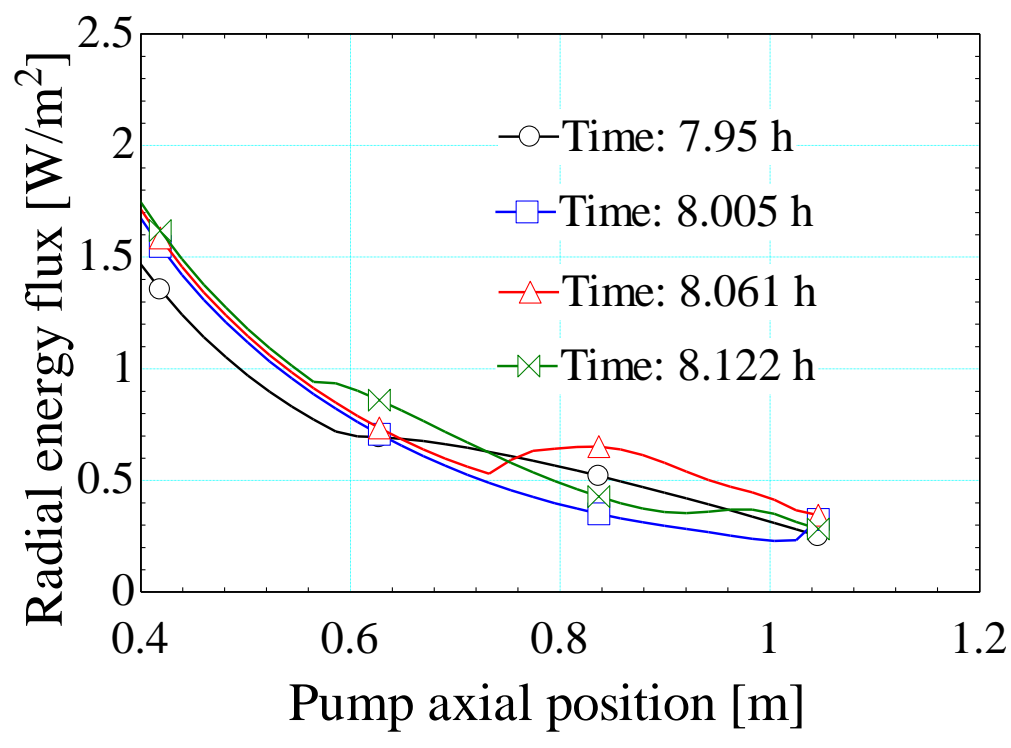

FIGURE 17b. Radial energy flux as a function of pump axial position. 


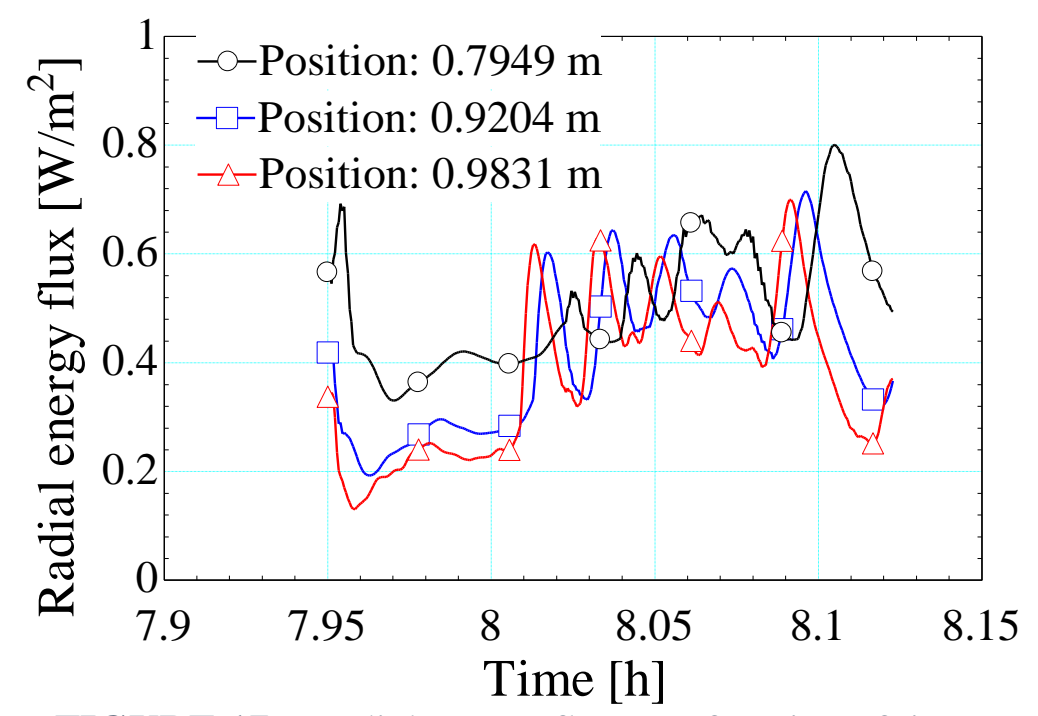

FIGURE 17c. Radial energy flux as a function of time.

As a final observation, Figures $18 \mathrm{a}$ and $18 \mathrm{~b}$ display the axial flux of the hydrogen gas, reflecting a reduction in axial flux in the same region where the radial flux is nonzero in keeping with an overall mass flow balance.

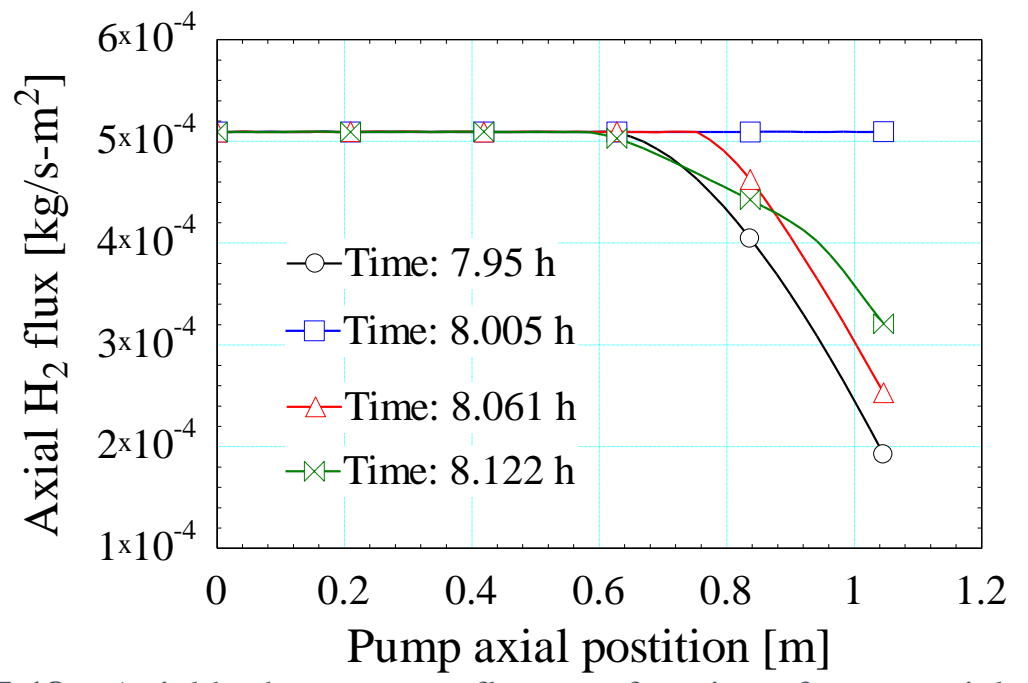

FIGURE 18a. Axial hydrogen mass flux as a function of pump axial position. 


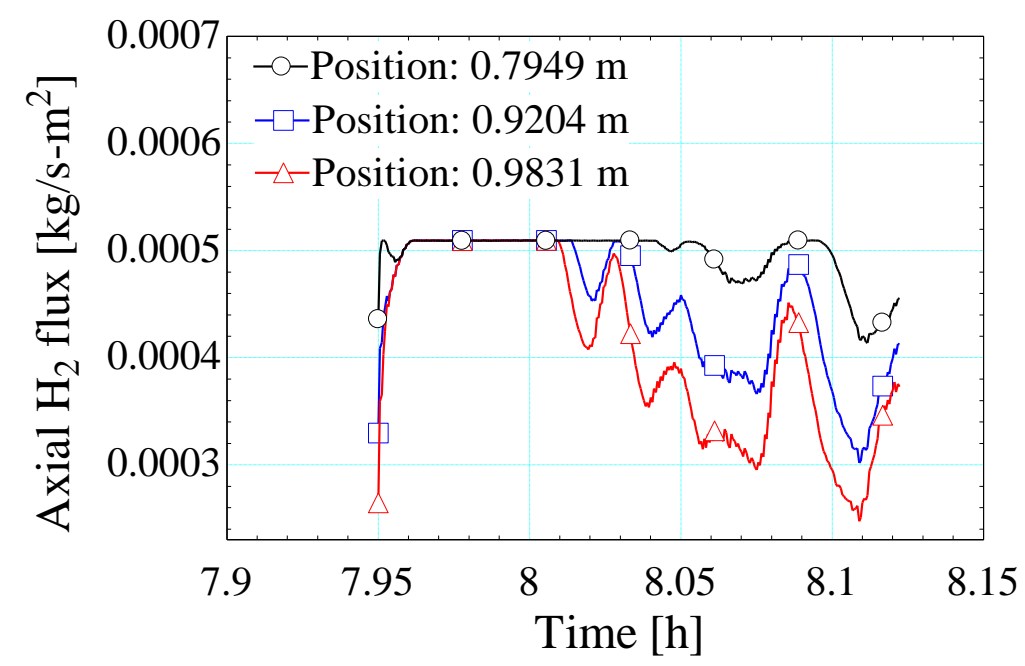

FIGURE 18b. Axial hydrogen mass flux as a function of time.

\subsection{Hydrogen pressure and adsorption}

As displayed in the above results, the temperature of the pump wall strongly influences the pumping performance of the CVC. The helium coolant cools the pump wall to sufficiently low temperatures, and the cold wall adsorbs hydrogen molecules. As a result, it can be appropriately named a cryogenic pump.

As also shown in the experimental data, the inlet hydrogen gas pressure is an important indicator for the pumping performance. Without any adsorption or sublimation of the hydrogen molecules, the inlet hydrogen gas pressure remains constant. With adsorption or sublimation, the inlet hydrogen gas pressure varies with time. Figure 3 demonstrates that the vapor pressure of hydrogen decreases dramatically with a decrease of temperature. When the vapor pressure associated with a surface temperature is smaller than the pressure of some adjacent hydrogen gas, the hydrogen molecules in the hydrogen gas are adsorbed onto the pump surface. Without maintaining an equivalent supply of gas molecules, the adsorption of the hydrogen molecules noticeably reduces the hydrogen gas pressure. An exchange of molecules between the gas and the surface will then occur until an equilibrium pressure is reached. The inlet hydrogen pressure approaches the equilibrium pressure, and the difference drives the deposition.

A comparison between the inlet hydrogen gas pressure and the hydrogen vapor pressure corresponding to the coldest wall temperature is presented in Figure 19. The inlet hydrogen gas pressure (the upstream hydrogen pressure in the experiment) is measured by the pressure gauge that is mounted with a capillary tube located $7 \mathrm{~cm}$ from the left hand end of the pump. The hydrogen vapor pressure is the vapor-solid phase equilibrium pressure, based on the minimum pump wall temperature that is obtained from the transient model. The mathematical relationship between the hydrogen vapor pressure and the temperature is interpolated from the data shown in Figure 3. As Figure 19 reveals, the inlet hydrogen gas pressure does follow the hydrogen vapor pressure. In spite of this agreement, the model used the reported measurements of the hydrogen pressure as one of the key input pieces of information. In the future it should be possible 
to reduce the number of inputs by calculating the inlet hydrogen vapor pressure based on the minimum pump wall temperature.

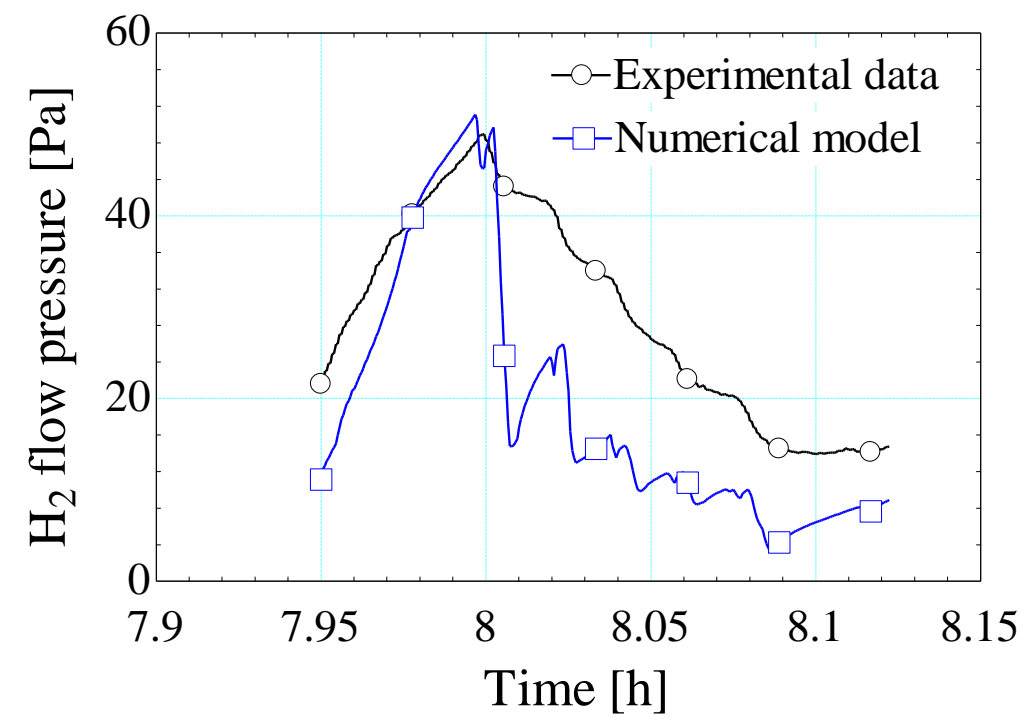

FIGURE 19. Measured inlet hydrogen gas pressure and the calculated hydrogen vapor pressure as a function of time. 


\section{Conclusions}

Using a 1-D numerical model based on the combined equations for conservation of species (mass), momentum, energy, as well as the ideal gas equation of state, and a unique solving approach called the Group-Member technique, we have been able to characterize the performance of a subscale test of the ITER cryoviscous compressor. Anticipated flow conditions within the pump create the unique situation for a cryo-pump in that the flow throughout the cryopump is viscous rather than molecular. In fact, as long as the wall temperature remains above $6.155 \mathrm{~K}$, the flow remains in the viscous regime.

The results of the model agree well with measurements taken at Oak Ridge National Lab wherein a portion of a flow stream of hydrogen gas flowing through a helium-cooled pipe is adsorbed onto the walls. Most significantly, the model allows one to identify the location and rate of hydrogen adsorption, and highlights the strong sensitivity of that process to the inlet temperature of the helium coolant. Radial mass flux rates of hydrogen on the order of $1 \times 10^{-5} \mathrm{~kg} / \mathrm{s}-\mathrm{m}^{2}$ are calculated to occur within the last $0.2 \mathrm{~m}$ of the axial pump length. Additionally, the model enables a full description of the pump wall temperature profile as a function of time.

In the specific experimental conditions to which the model results are compared, the single-component hydrogen gas exists in the viscous flow regime throughout the pump length. The model is also well suited to characterize the pumping performance of the CVC with gas mixtures of hydrogen-helium. Hydrogen-helium gas mixtures with anticipated helium concentrations of $1 \%$ and a total pressure as low as $10 \mathrm{~Pa}$ still provide viscous flow conditions.

The general formulation of the model allows a general applicability to other similar problems such as solid formation in cryogenic carbon capture systems, and ice build-up as a result of air-ingress in a cryogenic loss-of-vacuum situation.

\section{Acknowledgements}

This work is supported by the United States Department of Energy under contract number DE-FG02-05ER54821. The authors wish to thank Larry Baylor and Robert Duckworth at Oak Ridge National Laboratory for their cooperation on this project.

\section{References}

[1] Pearce, R.J.H., Antipenkov, A., Bersier, J.L., Boussier, B., Bryan, S., Dremel, M., Hughes, S., Sekachev, I., Worth, L., Baylor, L., Gardner, W., Meitner, S., Wikus, P., Laesser, R., and Papastergiou, S., "Gas species, their evolution and segregation through the ITER vacuum system," Vacuum, vol. 86, pp. 1725-1730, (2012).

[2] Day, C., Brennan, D., Camp, P., Jensen, H.S., Jones, G., Mack, A, and Miller, A. "Performance of ITER-Relevant Cryopump Panels for Tritiated Gases," 7th Tritium Science and Technology Conference, Baden Baden, Germany, EFDAJET-CP(04)05-04, pp. 29-34. (2005). 
[3] Day, C. Murdoch, D, and Pearce, R., "The vacuum systems of ITER." Vacuum vol. 83, pp. 773-778, (2009).

[4] Baylor, L.M., Barbier, C., Combs, S., Duckworth, R., Edgemon, T., Fehling, D. R., Boisson, J., "Cryogenic viscous compressor development and modeling for the ITER vacuum system," Proceedings from the $24^{\text {th }}$ Symposium on Fusion Engineering (SOFE), 2011 IEEE/NPSS pp. 1-4. (2011)

[5] Duckworth, R. C., Baylor, L. R., Meitner, S. J., Combs, S. K., Rasmussen, D. A., Hechler, M., Boissin, J.C. "Development and demonstration of a supercritical helium-cooled cryogenic viscous compressor prototype for the ITER vacuum system," Advances in Cryogenic Engineering vol. 57, pp. 1234-1242, (2012).

[6] Duckworth, R.C. private communication - details of the subscale CVC test.

[7] Zhang, D.S., Miller, F.K., and Pfotenhauer, J.M., "Modeling results for the ITER Cryogenic Fore Pump," Advances in Cryogenic Engineering, vol 59A, pp. 618625. (2014)

[8] Hands, B. "Introduction to cryopump design," Vacuum, vol. 26, pp. 11-16, (1976)

[9] Bentley, P., "The modern cryopump," Vacuum, vol. 30, pp. 145-158, (1980).

[10] Luo, X., Day, C., Haas, H., Varoutis, S., "Experimental results and numerical modeling of a high-performance large-scale cryopump. I. Test particle Monte Carlo simulation," Journal of Vacuum Science \& Technology A: Vacuum, Surfaces, and Films, vol. 29(4), pp. 041601-1- 041601-7 (2011).

[11] Varoutis, S., Day, C., "Follow-up vacuum study of the ITER model cryopump by the direct simulation Monte Carlo method," Verhandlungen der Deutschen Physikalischen Gesellschaft. (2011).

[12] Varoutis, S., Day, C., "Numerical modeling of an ITER type Cryopump." Fusion Engineering and Design, vol. 87(7-8), pp. 1395-1398, (2012).

[13] Roder, H. M., Childs, G. E., McCarty, R. D., \& Angerhofer, P. E. (1973). Survey of the properties of the hydrogen isotopes below their critical temperatures. NBS Technical Report: NBS-TN-641, National Bureau of Standards, Boulder, Co. (1973).

[14] McCarty, R. D., Hord, J., Roder, H.M., Selected properties of hydrogen (engineering design data). NBS Monograph 168, National Engineering Lab. (NBS), Boulder Co. (1981)

[15] Tzemos, G., Gegenheimer, M., and Hood, C.B., "Design and Performance of a Liquid Helium Cryopump Operating in the Viscous Flow Regime." Advances in Cryogenic Engineering, vol. 31, pp. 575-581, (1986).

[16] Dwivedi, P., Gaur, V., Sharma, A., and Verma, N., "Comparative study of removal of volatile organic compounds by cryogenic condensation and adsorption by activated carbon fiber. Separation and Purification Technology, vol 39(1-2), pp. 23-37 (2004). 
[17] Nastaj, J.F., Ambrozek, B., Rudnicka, J., "Simulation studies of a vacuum and temperature swing adsorption process for the removal of VOC from waste air streams," International communications in heat and mass transfer, vol. 33, pp. 80-86, (2006).

[18] Munakataa, K., Beloglazov, S., Bekris, N., Glugla, M., Wagner, R., and Fanghanel, E., "Experimental and simulation study on adsorption of hydrogen isotopes on MS5A at 77K," Fusion Engineering and Design, vol. 82, pp. 2303-2310, (2007).

[19] Reid, R., Prausnitz, J., \& Poling, B., The properties of gases and liquids. New York, NY: McGraw Hill Book Co. (1987).

[20] Edwards, M.F., Richardson, J.F., "Gas dispersion in packed beds," Chemical Engineering Science, vol. 23, pp. 109-123, (1968).

[21] Wakao, N., Funazkri, T., "Effect of fluid dispersion coefficients on particle-to-fluid mass transfer coefficients in packed beds: Correlation of Sherwood numbers," Chemical Engineering Science, vol. 33, pp. 1375-1384, (1978).

[22] Schork, J.M., Fair, J.R., "Parametric analysis of thermal regeneration of adsorption beds. Industrial \& engineering chemistry research, vol. 27(3), pp. 457-469, (1988).

[23] Coulson, J.M., Chemical Engineering, Vol. 1. Pergamon Press (1990).

[24] Yang, R. Gas Separation by Adsorption Processes. UK: Imperial College Press (1997).

[25] Furry, W.H., Jones, R.C., and Onsager, L., "On the theory of isotope separation by thermal diffusion," Physical Review, vol. 55, pp. 1083-1095, (1939).

[26] Klein, S.A., Engineering equation solver-EES, F-Chart software, Madison, WI, (2015), see http://fchart.com/ees/.

[27] Nellis, G.F., Klein, S.A., Heat Transfer, Cambridge University Press, NY. (2008). 


\begin{tabular}{ccccccc}
\hline $\begin{array}{c}\text { Temperature } \\
{[\mathbf{K}]}\end{array}$ & 5 & 6 & 7 & 8 & 9 & 10 \\
$\begin{array}{c}\text { Vapor pressure } \\
{[\mathbf{P a}]}\end{array}$ & 0.00476 & 0.16 & 2.08 & 14.91 & 71.23 & 255.58 \\
\hline
\end{tabular}

Table 1. Hydrogen vapor pressure as a function of temperature 